\title{
Analytical solution of non-isothermal two-dimensional general rate model of liquid chromatography
}

\author{
Ugochukwu David Uche ${ }^{1,2} \cdot$ Shamsul Qamar ${ }^{1,3}$ (D) Andreas Seidel-Morgenstern ${ }^{3}$
}

Received: 13 March 2019 / Revised: 24 July 2019 / Accepted: 10 August 2019 / Published online: 26 August 2019

(c) The Author(s) 2019

\begin{abstract}
A non-isothermal two-dimensional general rate model is formulated and analytically solved to analyze the effects of temperature changes inside liquid chromatographic columns of cylindrical geometry. The model equations form a system of convection-diffusion partial differential equations. The finite-Hankel transformation, the Laplace transformation, the eigendecomposition technique and a conventional solution technique of ordinary differential equations are used to solve the equations of the model. The coupling between concentration and temperature fronts is demonstrated and important parameters that affect the performance of the column are evaluated. To find the ranges of validity of our analytical results, a semi-discrete high resolution finite volume method is applied to solve the same system of equations for both linear and nonlinear isotherms. The results of this contribution can be helpful to optimize non-isothermal liquid chromatographic processes in which both radial and axial gradients occur.
\end{abstract}

Keywords Non-isothermal chromatography $\cdot$ Linearized isotherm $\cdot$ Two-dimensional general rate model $\cdot$ Analytical solutions $\cdot$ Finite volume scheme

\section{Introduction}

Mathematical modeling has contributed significantly in the advancements of liquid chromatography. Different researchers, involved in this field, have shown that vital information about the chromatographic process can be obtained through this approach (Guiochon 2002; Guiochon et al. 2006; Bellot and Condoretm 1991). Several mathematical models, characterized by different levels of complexities, have been introduced in the literature for describing liquid chromatography processes (Guiochon 2002; Guiochon et al. 2006; Guiochon and Lin 2003; Carta 1988; Ruthven 1984; Qamar et al. 2016, 2017).

In the non-isothermal liquid chromatography, several experimental studies have been carried out in the literature.

Shamsul Qamar

qamar@mpi-magdeburg.mpg.de

1 Department of Mathematics, COMSATS University Islamabad, Islamabad, Pakistan

2 Department of Mathematics Programme, National Mathematical Centre Abuja, Abuja, Nigeria

3 Max Planck Institute for Dynamics of Complex Technical Systems, Magdeburg, Germany
The earliest documented analysis of thermal effects was carried out to study deviation from linear chromatography by changing the sizes and concentrations of the injected pulses (Cerro and Smith 1969). Although less works have been done in this area, several contributions can still be found in the literature (Haynes 1986; Zhong and Meunier 1994; Sainio 2005; Sainio et al. 2007, 2011; Vu and Seidel-Morgenstern 2011; Javeed et al. 2012; Qamar et al. 2016).

This article extends the previous analysis in Qamar et al. (2017) to single-solute two-dimensional (2D) general rate model (GRM) of linear non-isothermal liquid chromatography. The contribution of this work include quantification of temperature effects on the concentration, demonstration of the concentration and thermal waves joint occurrence and identification of the main parameters that influence the temperature gradients in non-isothermal liquid chromatography. The equations of the model are solved analytically by simultaneously applying the Hankel transformation, the Laplace transformation, the eigen-decomposition technique, and a conventional technique for the solutions of ordinary differential equations (ODEs). The derived solutions are transformed back to their original coordinates numerically because they involve some complicated functions (Durbin 1974; Rice and Do 1995). Keeping in view the usefulness 
of temporal moments (Qamar et al. 2017; Suzuki 1973; Miyabe 2009; Qamar et al. 2014; Leweke and von Lieres 2016; Parveen et al. 2016; David et al. 2018), we numerically calculate the moments, as the analytical expressions of temporal moments are difficult to calculate. Analysis of the effects of different kinetic and thermodynamic parameters can be done by using these moments. To find the validity range of analytical results, we use a reliable finite volume scheme to approximate the equations of the model for nonlinear isotherm (David et al. 2018; Koren 1993; Javeed et al. 2011). Case studies, which cover a wide range of kinetic and thermodynamic parameters, are carried out to illustrate the potential of our analytical solutions. The results obtained are useful for further developments in the non-isothermal liquid chromatographic processes. For instance, the current analysis can be used (i) to study the effects of mass transfer kinetics on the elution profiles, (ii) for sensitivity analysis, (iii) for validating numerical solutions, and (iv) for determining longitudinal and radial dispersion coefficients from experimentally determined elution profiles, among others.

The current 2D-model equations and the derived analytical solutions are useful when a radial profile is introduced at the inlet of the column at the time of injection and when the radial temperature gradients are occurring in the column together with the radial concentration gradients. These scenarios could happen when (a) injection at the inlet of the column is imperfect, (b) ratio of the column radius to its length is larger, and (c) the column is not homogeneously packed. In many processes of liquid chromatography, the results of simple 1D-models deviates more or less strongly from predictions, however, these differences are difficult to quantify. Thus, finding a quantitative tool and a selection criteria for the right model becomes necessary. With the present nonisothermal 2D-GRM, the aforementioned situations can be studied by the assuming the inner or outer zones injections.

In summary, this work can be considered as a first initiative for the simulation of such a complicated process. No experimental validation is currently available for such a truly 2D-problem. However, this can be made possible in future by exploiting a dedicated injection system (to be designed and incorporated) utilizing only parts of the inlet cross-sectional area.

This article is further arranged as follows. In Sect. 2, a 2D-GRM is formulated to simulate dynamical processes in the non-isothermal liquid chromatographic columns of cylindrical geometry. In Sect. 3, analytical solutions of the non-isothermal 2D-GRM are derived for Dirichlet and Danckwerts boundary conditions (BCs). Section 4 presents some numerical test problems followed by the conclusions in Sect. 5.

\section{The non-isothermal 2D-GRM}

Consider a non-isothermal adsorption column packed with spherical shaped fully-porous particles having radius $R_{p}$. We take $t, z$ and $r$ to represent the time, axial and radial coordinates, respectively. Two specific conditions of injection are considered for triggering the radial mass and heat transfer effects. A new parameter $\tilde{r}$ is introduced for dividing the inlet cross sectional area of the column into an inner cylindrical core and into an outer annular ring, see Qamar et al. (2014, 2017) and David et al. (2018). Thus, sample can be injected to the column either through an inner, through an outer ring, or through the whole cross section. The latter case is possible when $\tilde{r}$ is set equal to the radius of the column denoted by $R_{c}$. The classical equation of mass balance for a single-solute in the bulk phase of the fluid is given as Qamar et al. (2014, 2017), David et al. (2018)

$$
\begin{aligned}
\epsilon_{b} & \frac{\partial c}{\partial t}+\left(1-\epsilon_{b}\right) \frac{\partial \bar{q}}{\partial t}+\epsilon_{b} u \frac{\partial c}{\partial z} \\
& =\epsilon_{b} D_{z} \frac{\partial^{2} c}{\partial z^{2}}+\epsilon_{b} D_{r}\left(\frac{\partial^{2} c}{\partial r^{2}}+\frac{1}{r} \frac{\partial c}{\partial r}\right) .
\end{aligned}
$$

In the equation above, $c$ represents the solute concentration in the bulk phase of the fluid, $\bar{q}$ represents averaged concentration in the solid particles, $\epsilon_{b}$ is the external porosity, $u$ represents the velocity, $D_{z}$ denotes the dispersion coefficient in the axial direction and $D_{r}$ denotes the dispersion coefficient in the radial direction. The averaged concentration in the spherical solid particles of volume $V_{p}$ and radius $R_{P}$ is defined as

$\bar{q}=\frac{1}{V_{p}} \int_{0}^{V_{p}} \mathrm{q}(\mathrm{V}) \mathrm{dV}=\frac{3}{\mathrm{R}_{\mathrm{p}}^{3}} \int_{0}^{\mathrm{R}_{\mathrm{p}}}\left[\epsilon_{\mathrm{p}} \mathrm{c}_{\mathrm{p}} \mathrm{r}_{\mathrm{p}}^{2}+\left(1-\epsilon_{\mathrm{b}}\right) \mathrm{q}_{\mathrm{p}} \mathrm{r}_{\mathrm{p}}^{2}\right] \mathrm{dr}_{\mathrm{p}}$,

where $\epsilon_{p}$ is the internal particle porosity, $c_{p}$ is the solute concentration in the particle pores and $q_{p}$ is the local equilibrium concentration of solute in stationary phase. Thus, for the time derivative of Eq. (2), considering the external mass transfer resistance, holds

$\frac{\partial \bar{q}}{\partial t}=\frac{3}{R_{p}} k_{\text {ext }}\left(c-\left.c_{p}\right|_{r_{p}=R_{p}}\right)$,

where $r_{p}$ represents the radial coordinate with spherical particles of radius $R_{p}$ and $k_{\text {ext }}$ denotes external mass transfer coefficient.

The equation of mass for the solute concentration in the particles pores can be given as

$\epsilon_{p} \frac{\partial c_{p}}{\partial t}+\left(1-\epsilon_{p}\right) \frac{\partial q_{p}}{\partial t}=\epsilon_{p} D_{p}\left[\frac{1}{r_{p}^{2}} \frac{\partial}{\partial r_{p}}\left(r_{p}^{2} \frac{\partial c_{p}}{\partial r_{p}}\right)\right]$, 
where, $D_{p}$ is the pore diffusivity.

The corresponding energy balance of the column is given as

$$
\begin{array}{r}
\epsilon_{b} \rho^{L} c_{P}^{L} \frac{\partial T_{b}}{\partial t}+\left(1-\epsilon_{b}\right) \overline{\rho c_{p}} \frac{\partial \bar{T}}{\partial t}+\epsilon_{b} \rho^{L} c_{P}^{L} u \frac{\partial T_{b}}{\partial z} \\
=\epsilon_{b} \lambda_{\mathrm{eff}, \mathrm{z}} \frac{\partial^{2} T_{b}}{\partial z^{2}}+\epsilon_{b} \lambda_{\mathrm{eff}, \mathrm{r}}\left(\frac{\partial^{2} T_{b}}{\partial r^{2}}+\frac{1}{r} \frac{\partial T_{b}}{\partial r}\right),
\end{array}
$$

where, $T_{b}$ is the bulk temperature, $\bar{T}$ is the averaged temperature of the particles, $\lambda_{\text {eff, } \mathrm{z}}$ represents the effective axial heat conductivity, $\lambda_{\text {eff,r }}$ denotes the effective radial heat conductivity coefficient, $\rho^{L}$ is the density per unit volume, and $c_{P}^{L}$ is the heat capacity for the liquid phase. Moreover,

$\overline{\rho c_{p}}=\epsilon_{p} \rho^{\mathrm{L}} \mathrm{c}_{\mathrm{P}}^{\mathrm{L}}+\left(1-\epsilon_{\mathrm{p}}\right) \rho^{\mathrm{S}} \mathrm{c}_{\mathrm{P}}^{\mathrm{S}}$.

Here, $\rho^{S}$ is the density and $c_{P}^{S}$ is the heat capacity of the solid phase. Furthermore, $\rho^{L}, \rho^{S}, c_{P}^{L}$ and $c_{P}^{S}$ are considered independent of temperature, which are valid in a limited temperature range.

The averaged particle temperature is defined as

$\bar{T}=\frac{3}{R_{p}} \int_{0}^{R_{p}} \mathrm{~T}_{\mathrm{P}} \mathrm{r}_{\mathrm{p}}^{2} \mathrm{dr}_{\mathrm{p}}$.

The time derivative of Eq. (7) is due to heat transport over the particle surface according to

$\overline{\rho c_{p}} \frac{\partial \bar{T}}{\partial t}=\frac{3}{R_{p}} h_{\mathrm{eff}}\left(T_{b}-\left.T_{p}\right|_{r_{p}=R_{p}}\right)$,

where, $h_{\text {eff }}$ is the effective particle to fluid heat transfer coefficient.

The radial temperature profile inside the spherical particles pores can be described by

$\overline{\rho c_{p}} \frac{\partial T_{p}}{\partial t}+\left(1-\epsilon_{p}\right)\left(-\Delta H_{A}\right) \frac{\partial q_{p}}{\partial t}=\epsilon_{p} \lambda_{p}\left[\frac{1}{r_{p}^{2}} \frac{\partial}{\partial r_{p}}\left(r_{p}^{2} \frac{\partial T_{p}}{\partial r_{p}}\right)\right]$,

where, $\Delta H_{A}$ represents the enthalpy of adsorption and $\lambda_{p}$ denotes the internal heat diffusivity coefficient.

The amount of absorbed solute is dependent on the temperature and is described by the enthalpy of adsorption by the van't Hoff relation. Therefore, the equilibrium relation in concentration is expressed as

$q_{p}\left(c_{p}, T_{p}\right)=a^{\mathrm{ref}} c_{p} \exp \left(\frac{-\Delta H_{\mathrm{A}}}{R_{\mathrm{g}}}\left(\frac{1}{T_{p}}-\frac{1}{T^{\mathrm{ref}}}\right)\right)$.
Here, $a_{\text {ref }}$ represents the equilibrium constant at the reference temperature $T^{\text {ref }}$ and $R_{\mathrm{g}}$ is the general gas constant.

In order to proceed, we define

$c_{1}=c, \quad c_{2}=T_{b}, \quad c_{p, 1}=c_{p}, \quad c_{p, 2}=T_{p}$.

By using Eq. (3) in Eq. (1), Eq. (8) in Eq. (5), Eq. (6) in Eq.

(9) and the above transformations in Eqs. (1), (4), (5) and (9), we get

$$
\begin{aligned}
\frac{\partial c_{1}}{\partial t}+u \frac{\partial c_{1}}{\partial z}= & D_{z} \frac{\partial^{2} c_{1}}{\partial z^{2}}+\epsilon_{b} D_{r}\left(\frac{\partial^{2} c_{1}}{\partial r^{2}}+\frac{1}{r} \frac{\partial c_{1}}{\partial r}\right) \\
& -F_{b} \frac{3}{R_{p}} k_{\text {ext }}\left(c_{1}-\left.c_{p, 1}\right|_{r_{p}=R_{p}}\right),
\end{aligned}
$$

$$
\begin{aligned}
\frac{\partial c_{p, 1}}{\partial t}+F_{p} \frac{\partial q_{p}}{\partial t} & =D_{\mathrm{p}}\left[\frac{1}{r_{p}^{2}} \frac{\partial}{\partial r_{p}}\left(r_{p}^{2} \frac{\partial c_{p, 1}}{\partial r_{p}}\right)\right], \\
\frac{\partial c_{2}}{\partial t}+u \frac{\partial c_{2}}{\partial z}= & \frac{\lambda_{\mathrm{eff}, \mathrm{z}}}{\rho^{L} c_{P}^{L}} \frac{\partial^{2} c_{2}}{\partial z^{2}}+\frac{\lambda_{e f f, r}}{\rho^{L} c_{p}^{L}}\left(\frac{\partial^{2} c_{2}}{\partial r^{2}}+\frac{1}{r} \frac{\partial c_{2}}{\partial r}\right) \\
& -\frac{3}{R_{p} \rho^{L} c_{P}^{L}} F_{b} h_{\mathrm{eff}}\left(c_{2}-\left.c_{p, 2}\right|_{r_{p}=R_{p}}\right),
\end{aligned}
$$

$$
\begin{gathered}
\left(1+\frac{F_{p} \rho^{s} c_{P}^{s}}{\rho^{L} c_{P}^{L}}\right) \frac{\partial c_{p, 2}}{\partial t}+\frac{\Delta H_{A} F_{p}}{\rho^{L} c_{P}^{L}} \frac{\partial q_{p}}{\partial t} \\
=\frac{\lambda_{p}}{\rho^{L} c_{P}^{L}}\left[\frac{1}{r_{p}^{2}} \frac{\partial}{\partial r_{p}}\left(r_{p}^{2} \frac{\partial c_{p, 2}}{\partial r_{p}}\right)\right] .
\end{gathered}
$$

Here, $F_{b}=\frac{1-\epsilon_{b}}{\epsilon_{b}}$ and $F_{p}=\frac{1-\epsilon_{p}}{\epsilon_{p}}$. The following dimensionless variables are used to reduce and simplify the model equations:

$$
\begin{aligned}
x & =\frac{z}{L}, \quad \tau=\frac{u t}{L}, \quad \rho=\frac{r}{R_{c}}, \quad \rho_{p}=\frac{r_{p}}{R_{p}}, \quad P e_{z}=\frac{L u}{D_{z}}, \\
P e_{\rho} & =\frac{R_{c}^{2} u}{D_{r} L} \quad P e_{z, T}=\frac{L u \rho^{L} c_{P}^{L}}{\lambda_{\mathrm{eff}, z}}, \\
P e_{\rho, T} & =\frac{\epsilon_{b} R_{c}^{2} u \rho^{L} c_{p}^{L}}{\lambda_{\mathrm{eff}, r} L}, \quad \zeta=\frac{k_{\mathrm{ext}} R_{p}}{D_{p}} \quad \zeta_{T}=\frac{h_{\mathrm{eff}} R_{p}}{\lambda_{p}}, \\
\eta & =\frac{D_{P} L}{R_{p}^{2} u}, \quad \eta_{T}=\frac{\lambda_{p} L}{R_{p}^{2} u \rho^{L} c_{P}^{L}}, \\
\xi & =3 \zeta \eta F_{b}, \quad \xi_{T}=3 \zeta_{T} \eta_{T} F_{b} .
\end{aligned}
$$

Here, $L$ is the characteristic column length, $P e_{z}$ and $P e_{z, T}$ are the axial peclet numbers, $P e_{\rho}$ and $P e_{\rho, T}$ are the radial peclet 
numbers, $\zeta$ and $\zeta_{T}$ are the Biot numbers for mass and energy, and $\eta, \eta_{T}, \xi$, and $\xi_{T}$ are the dimensionless constants. Using the above dimensionless variables in Eqs. (12)-(15), we get

$$
\begin{aligned}
& \frac{\partial c_{1}}{\partial \tau}+\frac{\partial c_{1}}{\partial x}=\frac{1}{P e_{z}} \frac{\partial^{2} c_{1}}{\partial x^{2}}+\frac{1}{P e_{\rho}}\left(\frac{\partial^{2} c_{1}}{\partial \rho^{2}}+\frac{1}{\rho} \frac{\partial c_{1}}{\partial \rho}\right) \\
& -\xi\left[c_{1}-\left.c_{p, 1}\right|_{\rho_{p}=1}\right] \text {, } \\
& \frac{\partial c_{p, 1}}{\partial \tau}+F_{p} \frac{\partial q_{p}}{\partial \tau}=\frac{\eta}{\rho_{p}^{2}} \frac{\partial}{\partial \rho_{p}}\left(\rho_{p}^{2} \frac{\partial c_{p, 1}}{\partial \rho_{p}}\right), \\
& \frac{\partial c_{2}}{\partial \tau}+\frac{\partial c_{2}}{\partial x}=\frac{1}{P e_{z, T}} \frac{\partial^{2} c_{2}}{\partial x^{2}}+\frac{1}{P e_{\rho, T}}\left(\frac{\partial^{2} c_{2}}{\partial \rho^{2}}+\frac{1}{\rho} \frac{\partial c_{2}}{\partial \rho}\right) \\
& -\xi_{T}\left[c_{2}-\left.c_{p, 2}\right|_{\rho_{p}=1}\right] \text {, } \\
& \left(1+F_{p} \frac{\rho^{S} c_{p}^{S}}{\rho^{L} c_{p}^{L}}\right) \frac{\partial c_{p, 2}}{\partial \tau}+\frac{\Delta H_{A} F_{p}}{\rho^{L} c_{p}^{L}} \frac{\partial q_{p}}{\partial \tau} \\
& =\frac{\eta_{T}}{\rho_{p}^{2}} \frac{\partial}{\partial \rho_{p}}\left(\rho_{p}^{2} \frac{\partial c_{p, 2}}{\partial \rho_{p}}\right) \text {. }
\end{aligned}
$$

We use the Taylor-expansion up to first order to linearize the equilibrium relation in Eq. (10) by assuming small changes in the temperature and concentration:

$$
\begin{aligned}
q_{p}\left(c_{p}, T_{p}\right) \approx & q_{p}\left(c^{\mathrm{ref}}, T^{\mathrm{ref}}\right)+\frac{\partial q_{p}}{\partial T_{p}\left(c^{\mathrm{ref}}, T^{\mathrm{ref}}\right)}\left(T_{p}-T^{\mathrm{ref}}\right) \\
& +\frac{\partial q_{p}}{\partial c_{p}}\left(c_{\left(\text {ref }, T^{\mathrm{ref}}\right)}\left(c_{p}-c^{\mathrm{ref}}\right)\right.
\end{aligned}
$$

The expression given in Eq. (21) above, is determined and further simplified as

$$
\begin{aligned}
q_{p}\left(c_{p, 1}, c_{p, 2}\right) & =a^{\mathrm{ref}} c_{p, 1} \\
& +a^{\mathrm{ref}} c^{\mathrm{ref}} \frac{\Delta H_{\mathrm{A}}}{R_{g}\left(T^{\mathrm{ref}}\right)^{2}} c_{p, 2}-a^{\mathrm{ref}} c^{\mathrm{ref}} \frac{\Delta H_{\mathrm{A}}}{R_{g} T^{\mathrm{ref}}} .
\end{aligned}
$$

Here, $a^{\text {ref }}, c^{\text {ref }}$ and $T^{\text {ref }}$ represent the adsorption coefficient at reference temperature, reference concentration and reference temperature, respectively.

The initial conditions for a regenerated column are given as

$$
\begin{aligned}
& c_{1}(\rho, x, 0)=0, \quad c_{2}(\rho, x, 0)=c_{2}^{\text {init }}, \\
& 0 \leq \rho \leq 1, \quad 0 \leq x \leq 1,
\end{aligned}
$$

$$
\begin{gathered}
c_{p, 1}\left(\rho_{p}, \rho, x, 0\right)=0, \quad c_{p, 2}\left(\rho_{p}, \rho, x, 0\right)=c_{p, 2}^{\text {init }}, \\
0 \leq \rho_{p} \leq 1, \quad 0 \leq \rho \leq 1, \quad 0 \leq x \leq 1 .
\end{gathered}
$$

Here, $c_{2}^{\text {init }}$ and $c_{p, 2}^{\text {init }}$ represent the initial bulk and particle temperatures, respectively.

The boundary conditions for Eqs. (17) and (19), which correspond to the nonporous walls of the column and the symmetry of radial profile, are used along the radial coordinate at $\rho=0$ and $\rho=1$ :

$\frac{\partial c_{j}(\rho=0, x, \tau)}{\partial \rho}=0, \quad \frac{\partial c_{j}(\rho=1, x, \tau)}{\partial \rho}=0, \quad j=1,2$.

For Eqs. (17) and (19), we apply the Dirichlet and Danckwerts boundary conditions considering two types of injections (inner region and outer region injections) along the axial coordinate, see Qamar et al. $(2014,2017)$ and David et al. (2018) for more details. We give Danckwerts BCs and provide conditions under which these BCs reduce to Dirichlet BCs. Let us define

$\tilde{\rho}=\tilde{r} / R_{c}$,

where $\tilde{r}$ divides the inlet cross sectional area of the column into inner and outer zones and $R_{c}$ denotes the radius of the column (Qamar et al. 2014, 2017; David et al. 2018).

For pulse injection as Danckwerts inlet boundary conditions via the inner region, they are expressed for $j=1,2$ as

$$
\begin{aligned}
& c_{j}(\rho, x=0, \tau)-\frac{1}{P e_{j}} \frac{\partial c_{j}(\rho, x=0, \tau)}{\partial x} \\
& \quad= \begin{cases}c_{j}^{\text {inj }}, & \text { if } 0 \leq \rho \leq \tilde{\rho} \text { and } 0 \leq \tau \leq \tau_{\text {inj }}, \\
0, & \tilde{\rho}<\rho \leq 1 \text { or } \tau>\tau_{\text {inj }} .\end{cases}
\end{aligned}
$$

While, for pulse injection as Danckwerts inlet boundary conditions via the outer region, they are expressed as

$$
\begin{aligned}
& c_{j}(\rho, x=0, \tau)-\frac{1}{P e_{j}} \frac{\partial c_{j}(\rho, x=0, \tau)}{\partial x} \\
& \quad= \begin{cases}c_{j}^{\text {inj }}, & \text { if } \tilde{\rho}<\rho \leq 1 \text { and } 0 \leq \tau \leq \tau_{\text {inj }}, \\
0, & 0 \leq \rho \leq \tilde{\rho} \text { or } \tau>\tau_{\text {inj }} .\end{cases}
\end{aligned}
$$

The Neumann boundary conditions at the outlet of a finite length column are considered:

$\left.\frac{\partial c_{j}(\rho, x, \tau)}{\partial x}\right|_{x=1}=0$

Here, $c_{1}^{\text {inj }}$ and $c_{2}^{\text {inj }}$ are the inlet concentration and bulk temperature, respectively. Further, $P e_{j}$ represents $P e_{z}$ and $P e_{z, T}$ for $j=1$ and $j=2$, respectively. The Dirichlet BCs are 
deduced from the Danckwerts BCs by letting $P e_{z} \rightarrow \infty$ and $P e_{z, T} \rightarrow \infty$ in Eqs. (27a) and (27b) and by replacing $x=\infty$ in Eq. $(27 \mathrm{c})$.

For Eqs. (18) and (20), the boundary conditions at $\rho_{p}=0$ and $\rho_{p}=1$ are expressed as

$$
\begin{aligned}
& \left.\frac{\partial c_{p, j}}{\partial \rho_{p}}\right|_{\rho_{p}=0}=0,\left.\quad \frac{\partial c_{p, j}}{\partial \rho_{p}}\right|_{\rho_{p}=1} \\
& =\zeta_{j}\left(c_{j}-\left.c_{p, j}\right|_{\rho_{p}=1}\right), \quad j=1,2 .
\end{aligned}
$$

Here, $\zeta_{j}$ represents $\zeta$ and $\zeta_{T}$ for $j=1$ and $j=2$, respectively.

\section{Analytical solutions}

The aforementioned model equations are solved applying first the Hankel transform, followed by Laplace transform, the decoupling technique and a conventional ODE solution technique. The Hankel transform of $c(\rho, x, \tau)$ is defined as (c.f. Carslaw and Jaeger 1953; Chen et al. 2011; Crank 1975; Sneddon 1972)

$$
\begin{aligned}
c_{H, j}\left(\lambda_{n}, x, \tau\right) & =H\left[c_{j}(\rho, x, \tau)\right] \\
& =\int_{0}^{1} c_{j}(\rho, x, \tau) J_{0}\left(\lambda_{n} \rho\right) \rho d \rho, \quad j=1,2 .
\end{aligned}
$$

After applying the above transformation on Eqs. (17) and (19) with respect to coordinate $\rho$, we obtain

$$
\begin{aligned}
\frac{\partial c_{H, 1}}{\partial \tau}+\frac{\partial c_{H, 1}}{\partial x}= & \frac{1}{P e_{z}} \frac{\partial^{2} c_{H, 1}}{\partial x^{2}}-\frac{\lambda_{n}^{2}}{P e_{\rho}} c_{H, 1} \\
& -\xi\left(c_{H, 1}-\left.c_{p H, 1}\right|_{\rho_{p}=1}\right), \\
\frac{\partial c_{H, 2}}{\partial \tau}+\frac{\partial c_{H, 2}}{\partial x}= & \frac{1}{P e_{z, T}} \frac{\partial^{2} c_{H, 2}}{\partial x^{2}}-\frac{\lambda_{n}^{2}}{P e_{\rho, T}} c_{H, 2} \\
& -\xi_{T}\left(c_{H, 2}-\left.c_{p H, 2}\right|_{\rho_{p}=1}\right) .
\end{aligned}
$$

Here, $c_{H, j}\left(\lambda_{n}, x, \tau\right)$ and $c_{p H, j}\left(\lambda_{n}, x, \tau\right)$ denote the zeroth-order finite Hankel transforms of $c_{j}(\rho, x, \tau)$ and $c_{p, j}\left(\rho_{p}, \rho, x, \tau\right)$, respectively. Thus, the Hankel transformation eliminates the radial derivatives from the partial differential equations (PDEs). Next, we apply the Laplace transform defined as (c.f Sneddon 1972)

$\bar{c}_{H, j}\left(\lambda_{n}, x, s\right)=\int_{0}^{\infty} e^{-s t} c_{H, j}\left(\lambda_{n}, x, \tau\right) d t, \quad t \geq 0$, on Eqs. (29) and (30) with respect to $\tau$ for zero initial concentration of the solute to obtain

$$
\begin{gathered}
\frac{1}{P e_{z}} \frac{\partial^{2} \bar{c}_{H, 1}}{\partial x^{2}}-\frac{\partial \bar{c}_{H, 1}}{\partial x}-\left(s+\frac{\lambda_{n}^{2}}{P e_{\rho}}\right) \bar{c}_{H, 1} \\
-\xi\left(\bar{c}_{H, 1}-\left.\bar{c}_{p H, 1}\right|_{\rho_{p}=1}\right)=0
\end{gathered}
$$

$$
\begin{aligned}
& \frac{1}{P e_{z, T}} \frac{\partial^{2} \bar{c}_{H, 2}}{\partial x^{2}}-\frac{\partial \bar{c}_{H, 2}}{\partial x}-\left(s+\frac{\lambda_{n}^{2}}{P e_{\rho, T}}\right) \bar{c}_{H, 2} \\
& -\xi_{T}\left(\bar{c}_{H, 2}-\left.\bar{c}_{p H, 2}\right|_{\rho_{p}=1}\right)=0 .
\end{aligned}
$$

Subsequently, applying the Hankel and Laplace transformations on the Danckwerts boundary conditions given in Eqs. (27a) and (27c), we obtain

$$
\begin{gathered}
c_{H, j}\left(\lambda_{n}, x=0, \tau\right)-\frac{1}{P e_{j}} \frac{\partial c_{H, j}\left(\lambda_{n}, x=0, \tau\right)}{\partial x} \\
= \begin{cases}c_{j}^{\text {inj }} F\left(\lambda_{n}\right), & \text { if } 0 \leq \tau \leq \tau_{\text {inj }}, \\
0, & \text { if } \tau>\tau_{\text {inj }},\end{cases}
\end{gathered}
$$

$\left.\frac{\partial c_{H, j}\left(\lambda_{n}, x, \tau\right)}{\partial x}\right|_{x=1}=0, \quad j=1,2$,

and

$$
\begin{gathered}
\bar{c}_{H, j}\left(\lambda_{n}, x=0, s\right)-\frac{1}{P e_{j}} \frac{\partial \bar{c}_{H, j}\left(\lambda_{n}, x=0, s\right)}{\partial x} \\
=\frac{c_{j}^{\text {inj }} F\left(\lambda_{n}\right)}{s}\left(1-e^{-s \tau_{\text {inj }}}\right),
\end{gathered}
$$

$\left.\frac{\partial \bar{c}_{H, j}}{\partial x}\right|_{x=1}=0, \quad j=1,2$.

Here, $\bar{c}_{H, j}$ denotes the concentration after Hankel and Laplace transformation for $j=1$ and temperature for $j=2$, respectively.

Injecting through the inner region, $F\left(\lambda_{n}\right)$ is given as

$F\left(\lambda_{n}\right)= \begin{cases}\frac{\tilde{\rho}^{2}}{2}, & \text { if } \lambda_{n}=0, \\ \frac{\hat{\rho}}{\lambda_{n}} J_{1}\left(\lambda_{n} \tilde{\rho}\right), & \text { if } \lambda_{n} \neq 0,\end{cases}$

and injecting through the outer region, $F\left(\lambda_{n}\right)$ is given as 
$F\left(\lambda_{n}\right)= \begin{cases}\left(\frac{1}{2}-\frac{\tilde{\rho}^{2}}{2}\right), & \text { if } \lambda_{n}=0, \\ -\frac{\tilde{\rho}}{\lambda_{n}} J_{1}\left(\lambda_{n} \tilde{\rho}\right), & \text { if } \lambda_{n} \neq 0 .\end{cases}$

Next, using Eq. (22) in Eqs. (18) and (20) and after rephrasing, we obtain

$\eta \frac{\partial^{2}}{\partial \rho_{p}^{2}}\left(\rho_{p} c_{p, 1}\right)=a_{1}^{*} \frac{\partial}{\partial \tau}\left(\rho_{p} c_{p, 1}\right)+a_{2}^{*} \frac{\partial}{\partial \tau}\left(\rho_{p} c_{p, 2}\right)$,

$\eta_{T} \frac{\partial^{2}}{\partial \rho_{p}^{2}}\left(\rho_{p} c_{p, 2}\right)=a_{3}^{*} \frac{\partial}{\partial \tau}\left(\rho_{p} c_{p, 1}\right)+a_{4}^{*} \frac{\partial}{\partial \tau}\left(\rho_{p} c_{p, 2}\right)$,

where

$$
\begin{aligned}
a_{1}^{*}= & \epsilon_{p}+a^{\mathrm{ref}}\left(1-\epsilon_{p}\right), \quad a_{4}^{*}=\epsilon_{p}+\left(1-\epsilon_{p}\right) \frac{\rho^{S} c_{p}^{S}}{\rho^{L} c_{p}^{L}} \\
& +\left(1-\epsilon_{p}\right)\left(a^{\mathrm{ref}} c^{\mathrm{ref}} \frac{\left(\Delta H_{\mathrm{A}}\right)^{2}}{R_{g} \rho^{L} c_{p}^{L}\left(T^{\mathrm{ref}}\right)^{2}}\right), \\
a_{2}^{*}= & \left(1-\epsilon_{p}\right)\left(a^{\mathrm{ref}} c^{\mathrm{ref}} \frac{\Delta H_{\mathrm{A}}}{R_{g}\left(T^{\mathrm{ref}}\right)^{2}}\right), \\
a_{3}^{*}= & \left(1-\epsilon_{p}\right)\left(a^{\mathrm{ref}} \frac{\Delta H_{\mathrm{A}}}{\rho^{L} c_{p}^{L}}\right) .
\end{aligned}
$$

After applying the Hankel and Laplace transforms on Eqs. (40) and (41), we obtain

$\frac{d^{2}}{d \rho_{p}^{2}}\left(\rho_{p} \bar{c}_{p H, 1}\right)=\alpha_{1}(s) \rho_{p} \bar{c}_{p H, 1}+\alpha_{2}(s) \rho_{p} \bar{c}_{p H, 2}$,

$\frac{d^{2}}{d \rho_{p}^{2}}\left(\rho_{p} \bar{c}_{p H, 2}\right)=\alpha_{3}(s) \rho_{p} \bar{c}_{p H, 1}+\alpha_{4}(s) \rho_{p} \bar{c}_{p H, 2}$,

where, $\alpha_{1}(s)=\frac{a_{1}^{*} s}{\eta}, \alpha_{2}(s)=\frac{a_{2}^{*} s}{\eta}, \alpha_{3}(s)=\frac{a_{3}^{*} s}{\eta_{T}}$, and $\alpha_{4}(s)=\frac{a_{4}^{*} s}{\eta_{T}}$.

Representing Eqs. (43) and (44) in matrix notation, we obtain

$\frac{d^{2}}{d \rho_{p}^{2}}\left(\begin{array}{c}\rho_{p} \bar{c}_{p H, 1} \\ \rho_{p} \bar{c}_{p H, 2}\end{array}\right)=\left(\begin{array}{cc}\alpha_{1}(s) & \alpha_{2}(s) \\ \alpha_{3}(s) & \alpha_{4}(s)\end{array}\right)\left(\begin{array}{c}\rho_{p} \bar{c}_{p H, 1} \\ \rho_{p} \bar{c}_{p H, 2}\end{array}\right)$.

We have a coupled system of equations above and the eigendecomposition technique is used to decouple the system in order to find the solutions by using the boundary conditions in Eq. (27d). Hence, Let $B$ represent the coefficient matrix of the system as
$B=\left(\begin{array}{cc}\alpha_{1}(s) & \alpha_{2}(s) \\ \alpha_{3}(s) & \alpha_{4}(s)\end{array}\right)$.

The above matrix has two distinct eigenvalues which are given as

$$
\begin{aligned}
\lambda_{1,2}= & \frac{1}{2}\left(\alpha_{1}(s)+\alpha_{4}(s)\right) \\
& \pm \frac{1}{2} \sqrt{\left(\alpha_{1}(s)-\alpha_{4}(s)\right)^{2}+4 \alpha_{2}(s) \alpha_{3}(s)},
\end{aligned}
$$

and the corresponding distinct eigenvectors

$x_{1}=\left[\begin{array}{c}\lambda_{1}-\alpha_{4}(s) \\ \alpha_{3}(s)\end{array}\right], \quad x_{2}=\left[\begin{array}{c}\lambda_{2}-\alpha_{4}(s) \\ \alpha_{3}(s)\end{array}\right]$.

Therefore, based on the above eigenvalues, the transformation matrix $A$ is obtained as

$A=\left(\begin{array}{cc}\lambda_{1}-\alpha_{4}(s) & \lambda_{2}-\alpha_{4}(s) \\ \alpha_{3}(s) & \alpha_{3}(s)\end{array}\right)$.

Using the matrix $A$, we can formulate the following linear transformation as

$\left[\begin{array}{c}\bar{c}_{p H, 1} \\ \bar{c}_{p H, 2}\end{array}\right]=\left(\begin{array}{cc}\lambda_{1}-\alpha_{4}(s) & \lambda_{2}-\alpha_{4}(s) \\ \alpha_{3}(s) & \alpha_{3}(s)\end{array}\right)\left[\begin{array}{c}\bar{b}_{p H, 1} \\ \bar{b}_{p H, 2}\end{array}\right]$.

We use the linear transformation above on Eq. (45) to obtain

$\frac{d^{2}}{d \rho_{p}^{2}}\left(\begin{array}{c}\rho_{p} \bar{b}_{p H, 1} \\ \rho_{p} \bar{b}_{p H, 2}\end{array}\right)=\left(\begin{array}{cc}\lambda_{1} & 0 \\ 0 & \lambda_{2}\end{array}\right)\left(\begin{array}{c}\rho_{p} \bar{b}_{p H, 1} \\ \rho_{p} \bar{b}_{p H, 2}\end{array}\right)$.

Now, we have a system of ODEs in Eq. (51) with the following explicit solutions

$\bar{b}_{p H, 1}=\frac{1}{\rho_{p}}\left[C_{1} e^{\sqrt{\lambda_{1}} \rho_{p}}+C_{2} e^{-\sqrt{\lambda_{1}} \rho_{p}}\right]$,

$\bar{b}_{p H, 2}=\frac{1}{\rho_{p}}\left[D_{1} e^{\sqrt{\lambda_{2}} \rho_{p}}+D_{2} e^{-\sqrt{\lambda_{2}} \rho_{p}}\right]$.

From Eqs. (27d) and (50), we obtain the boundary conditions

$\left.\frac{\partial \bar{b}_{p H, 1}}{\partial \rho_{p}}\right|_{\rho_{p}=0}=0,\left.\quad \frac{\partial \bar{b}_{p H, 2}}{\partial \rho_{p}}\right|_{\rho_{p}=0}=0$.

By using the above boundary conditions, we get from Eq. (52) $C_{1}=-C_{2}$ and $D_{1}=-D_{2}$. Therefore, Eq. (52) reduces to

$\bar{b}_{p H, 1}=\frac{2 C_{1}}{\rho_{p}} \sinh \left(\sqrt{\lambda_{1}} \rho_{p}\right), \quad \bar{b}_{p H, 2}=\frac{2 D_{1}}{\rho_{p}} \sinh \left(\sqrt{\lambda_{2}} \rho_{p}\right)$.

By using the values of $\bar{b}_{p H, 1}$ and $\bar{b}_{p H, 1}$ in the transformation given by Eq. (50), we obtain the following results 


$$
\begin{aligned}
\bar{c}_{p H, 1}= & \left(\lambda_{1}-\alpha_{4}(s)\right)\left[\frac{2 C_{1}}{\rho_{p}} \sinh \left(\sqrt{\lambda_{1}} \rho_{p}\right)\right] \\
& +\left(\lambda_{2}-\alpha_{4}(s)\right)\left[\frac{2 D_{1}}{\rho_{p}} \sinh \left(\sqrt{\lambda_{2}} \rho_{p}\right)\right], \\
\bar{c}_{p H, 2}= & \alpha_{3}(s)\left[\frac{2 C_{1}}{\rho_{p}} \sinh \left(\sqrt{\lambda_{1}} \rho_{p}\right)\right] \\
& +\alpha_{3}(s)\left[\frac{2 D_{1}}{\rho_{p}} \sinh \left(\sqrt{\lambda_{2}} \rho_{p}\right)\right] .
\end{aligned}
$$

By using the boundary conditions of Eq. (27d) at $\rho_{p}=1$ in Eq. (55), we obtain

$$
\begin{aligned}
& C_{1}=-\frac{1}{2 \sinh \left(\sqrt{\lambda_{1}}\right)}\left[\frac{\phi_{1}(s)}{v(s)} \bar{c}_{H, 1}-\frac{\phi_{2}(s)}{v(s)} \bar{c}_{H, 2}\right], \\
& D_{1}=\frac{1}{2 \sinh \left(\sqrt{\lambda_{2}}\right)}\left[\frac{\phi_{3}(s)}{v(s)} \bar{c}_{H, 1}-\frac{\phi_{4}(s)}{v(s)} \bar{c}_{H, 2}\right],
\end{aligned}
$$

where

$$
\begin{aligned}
\phi_{1}(s)= & \zeta\left[\zeta_{T}-1+\sqrt{\lambda_{1}} \operatorname{coth}\left(\sqrt{\lambda_{1}}\right)\right], \\
\phi_{2}(s)= & \frac{\left(\lambda_{1}-\alpha_{4}(s)\right)}{\alpha_{3}(s)} \zeta_{T}\left[\zeta-1+\sqrt{\lambda_{1}} \operatorname{coth}\left(\sqrt{\lambda_{1}}\right)\right], \\
\phi_{3}(s)= & \zeta\left[\zeta_{T}-1+\sqrt{\lambda_{2}} \operatorname{coth}\left(\sqrt{\lambda_{2}}\right)\right], \\
\phi_{4}(s)= & \frac{\left(\lambda_{2}-\alpha_{4}(s)\right)}{\alpha_{3}(s)} \zeta_{T}\left[\zeta-1+\sqrt{\lambda_{2}} \operatorname{coth}\left(\sqrt{\lambda_{2}}\right)\right], \\
v(s)= & \left(\lambda_{2}-\alpha_{4}(s)\right)\left[\zeta-1+\sqrt{\lambda_{2}} \operatorname{coth}\left(\sqrt{\lambda_{2}}\right)\right]\left[\zeta_{T}-1\right. \\
& \left.+\sqrt{\lambda_{1}} \operatorname{coth}\left(\sqrt{\lambda_{1}}\right)\right] \\
& -\left(\lambda_{1}-\alpha_{4}(s)\right)\left[\zeta-1+\sqrt{\lambda_{1}} \operatorname{coth}\left(\sqrt{\lambda_{1}}\right)\right]\left[\zeta_{T}-1\right. \\
& \left.+\sqrt{\lambda_{2}} \operatorname{coth}\left(\sqrt{\lambda_{2}}\right)\right] .
\end{aligned}
$$

By using these values in Eq. (55), we get

$$
\begin{aligned}
\left.\bar{c}_{p H, 1}\right|_{\rho_{p}=1}= & {\left[\left(\lambda_{2}-\alpha_{4}(s)\right) \frac{\phi_{3}(s)}{v(s)}\right.} \\
& \left.-\left(\lambda_{1}-\alpha_{4}(s)\right) \frac{\phi_{1}(s)}{v(s)}\right] \bar{c}_{H, 1} \\
& +\left[\left(\lambda_{1}-\alpha_{4}(s)\right) \frac{\phi_{2}(s)}{v(s)}-\left(\lambda_{2}-\alpha_{4}(s)\right) \frac{\phi_{4}(s)}{v(s)}\right] \bar{c}_{H, 2},
\end{aligned}
$$

$$
\begin{gathered}
\left.\bar{c}_{p H, 2}\right|_{\rho_{p}=1}=\alpha_{3}(s)\left[\frac{\phi_{3}(s)}{v(s)}-\frac{\phi_{1}(s)}{v(s)}\right] \bar{c}_{H, 1} \\
+\alpha_{3}(s)\left[\frac{\phi_{2}(s)}{v(s)}-\frac{\phi_{4}(s)}{v(s)}\right] \bar{c}_{H, 2} .
\end{gathered}
$$

Putting the values of Eqs. (59) and (60) in Eqs. (32) and (33), we get the system of equations

$$
\begin{array}{r}
\frac{d^{2}}{d x^{2}}\left(\begin{array}{c}
\bar{c}_{H, 1} \\
\bar{c}_{H, 2}
\end{array}\right)-\left(\begin{array}{c}
P e_{z} \\
P e_{z, T}
\end{array}\right) \frac{d}{d x}\left(\begin{array}{c}
\bar{c}_{H, 1} \\
\bar{c}_{H, 2}
\end{array}\right) \\
=\left(\begin{array}{cc}
\gamma_{1}\left(s, \lambda_{n}\right) & \psi_{2}(s) \\
\psi_{3}(s) & \gamma_{2}\left(s, \lambda_{n}\right)
\end{array}\right)\left(\begin{array}{c}
\bar{c}_{H, 1} \\
\bar{c}_{H, 2}
\end{array}\right) .
\end{array}
$$

Here,

$\gamma_{1}\left(s, \lambda_{n}\right)=P e_{z}\left(s+\frac{\lambda_{n}^{2}}{P e_{\rho}}\right)+\psi_{1}(s)$,

$\gamma_{2}\left(s, \lambda_{n}\right)=P e_{z, T}\left(s+\frac{\lambda_{n}^{2}}{P e_{\rho, T}}\right)+\psi_{4}(s)$

and

$\psi_{1}(s)=P e_{z} \xi\left[1-\left(\lambda_{2}-\alpha_{4}(s)\right) \frac{\phi_{3}(s)}{v(s)}+\left(\lambda_{1}-\alpha_{4}(s)\right) \frac{\phi_{1}(s)}{v(s)}\right]$,

$\psi_{2}(s)=-P e_{z} \xi\left[\left(\lambda_{1}-\alpha_{4}(s)\right) \frac{\phi_{4}(s)}{v(s)}-\left(\lambda_{2}-\alpha_{4}(s)\right) \frac{\phi_{2}(s)}{v(s)}\right]$,

$\psi_{3}(s)=-\alpha_{3}(s) P e_{z, T} \xi_{T}\left[\frac{\phi_{3}(s)}{v(s)}-\frac{\phi_{1}(s)}{v(s)}\right]$,

$\psi_{4}(s)=P e_{z, T} \xi_{T}\left[1+\alpha_{3}(s)\left(\frac{\phi_{2}(s)}{v(s)}-\frac{\phi_{4}(s)}{v(s)}\right)\right]$.

In a similar manner, we decouple the system in Eq. (61) by using the following transformation

$\left[\begin{array}{c}\bar{c}_{H, 1} \\ \bar{c}_{H, 2}\end{array}\right]=\left(\begin{array}{cc}\lambda_{3}-\gamma_{2}\left(s, \lambda_{n}\right) & \lambda_{4}-\gamma_{2}\left(s, \lambda_{n}\right) \\ \psi_{3}(s) & \psi_{3}(s)\end{array}\right)\left[\begin{array}{l}\bar{b}_{H, 1} \\ \bar{b}_{H, 2}\end{array}\right]$,

where,

$$
\begin{aligned}
\lambda_{3,4}= & \frac{1}{2}\left(\gamma_{1}\left(s, \lambda_{n}\right)+\gamma_{2}\left(s, \lambda_{n}\right)\right) \\
& \pm \frac{1}{2} \sqrt{\left(\gamma_{1}\left(s, \lambda_{n}\right)-\gamma_{2}\left(s, \lambda_{n}\right)\right)^{2}+4 \psi_{2}(s) \psi_{3}(s)} .
\end{aligned}
$$

Thus, we get the form

$\frac{d^{2}}{d x^{2}}\left(\begin{array}{c}\bar{b}_{H, 1} \\ \bar{b}_{H, 2}\end{array}\right)-\left(\begin{array}{c}P e_{z} \\ P e_{z, T}\end{array}\right) \frac{d}{d x}\left(\begin{array}{c}\bar{b}_{H, 1} \\ \bar{b}_{H, 2}\end{array}\right)=\left(\begin{array}{cc}\lambda_{3} & 0 \\ 0 & \lambda_{4}\end{array}\right)\left(\begin{array}{c}\bar{b}_{H, 1} \\ \bar{b}_{H, 2}\end{array}\right)$.

The solutions of Eq. (69) are given as

$$
\begin{aligned}
\bar{b}_{H, 1}(x, s) & =C_{3} e^{m_{1} x}+C_{4} e^{m_{2} x}, \quad \bar{b}_{H, 2}(x, s) \\
& =D_{3} e^{n_{1} x}+D_{4} e^{n_{2} x} .
\end{aligned}
$$


Here,

$$
\begin{aligned}
m_{1,2} & =\frac{1}{2} P e_{z} \pm \frac{1}{2} \sqrt{P e_{z}^{2}-4 \lambda_{3}}, \\
n_{1,2} & =\frac{1}{2} P e_{z}, T \pm \frac{1}{2} \sqrt{P e_{z, T}^{2}-4 \lambda_{4}} .
\end{aligned}
$$

In Eq. (71), $m_{1}$ and $n_{1}$ are obtained by choosing the plus sign and $m_{2}$ and $n_{2}$ are obtained by considering the minus sign. Next, we use the two types of axial boundary conditions to find the integration constants $C_{3}, C_{4}, D_{3}$ and $D_{4}$.

By utilizing the transformation in Eq. (67), we can transform the boundary conditions in Eq. (70). After following a similar procedure of analytical solution derivation discussed in Qamar et al. (2017), we obtain the following HankelLaplace domains solutions for Dirichlet boundary conditions from Eqs. (36) and (37) for $P e_{i} \rightarrow \infty$ and $x=\infty$ as
Analytical Laplace and Hankel inversions are not possible for the above equations. Thus, to get back the solutions in the actual coordinates, we use the numerical Laplace and Hankel inversions (Durbin 1974). In the Appendix 1 of this manuscript, we have presented a detailed derivation of the Durbin method (Durbin 1974). The Matlab software was used to implement this technique.

Next, applying the transformation in Eq. (67) in a similar manner and following the solution procedure presented in Qamar et al. (2017), we obtain the following Hankel-Laplace domains solutions for the Danckwerts boundary conditions

$$
\begin{aligned}
& \bar{c}_{H, 1}\left(\lambda_{n}, x, s\right)= \frac{\left(1-e^{-s \tau_{\mathrm{inj}}}\right) c_{1}^{\mathrm{inj}} F\left(\lambda_{n}\right)}{\left(\lambda_{3}-\lambda_{4}\right) s}\left[\left(\lambda_{3}-\gamma_{2}\left(s, \lambda_{n}\right)\right) e^{m_{2} x}-\left(\lambda_{4}-\gamma_{2}\left(s, \lambda_{n}\right)\right) e^{n_{2} x}\right] \\
&+\frac{\left(1-e^{-s \tau_{\mathrm{inj}}}\right) c_{2}^{\mathrm{inj}} F\left(\lambda_{n}\right)\left(\lambda_{3}-\gamma_{2}\left(s, \lambda_{n}\right)\right)\left(\lambda_{4}-\gamma_{2}\left(s, \lambda_{n}\right)\right)}{\psi_{3}(s)\left(\lambda_{3}-\lambda_{4}\right) s}\left[e^{m_{2} x}-e^{n_{2} x}\right], \\
& \bar{c}_{H, 2}\left(\lambda_{n}, x, s\right)= \\
&+\frac{\left(1-e^{-s \tau_{\mathrm{inj}}}\right) \psi_{3}(s) c_{1}^{\mathrm{inj}} F\left(\lambda_{n}\right)}{\left(\lambda_{3}-\lambda_{4}\right) s}\left[e^{m_{2} x}-e^{n_{2} x}\right] \\
&+e^{\left.-s \tau_{\mathrm{inj}}\right) c_{2}^{\mathrm{inj}} F\left(\lambda_{n}\right)}\left[\left(\lambda_{4}-\gamma_{2}\left(s, \lambda_{n}\right)\right) e^{m_{2} x}-\left(\lambda_{3}-\gamma_{2}\left(s, \lambda_{n}\right)\right) e^{n_{2} x}\right] .
\end{aligned}
$$

$$
\begin{aligned}
& \bar{c}_{H, 1}\left(\lambda_{n}, x, s\right) \\
& =\frac{\left(1-e^{-s \tau_{\mathrm{inj}}}\right) c_{1}^{\mathrm{inj}} F\left(\lambda_{n}\right)}{\left(\lambda_{3}-\lambda_{4}\right) s}\left[v_{1}\left(\lambda_{3}-\gamma_{2}\left(s, \lambda_{n}\right)\right)\left(m_{1} e^{m_{2} x}-m_{2} e^{m_{1}(x-1)+m_{2}}\right)\right] \\
& -\frac{\left(1-e^{-s \tau_{\mathrm{inj}}}\right) c_{1}^{\mathrm{inj}} F\left(\lambda_{n}\right)}{\left(\lambda_{3}-\lambda_{4}\right) s}\left[v_{2}\left(\lambda_{4}-\gamma_{2}\left(s, \lambda_{n}\right)\right)\left(n_{1} e^{n_{2} x}-n_{2} e^{n_{1}(x-1)+n_{2}}\right)\right] \\
& -\frac{\left(1-e^{-s \tau_{\text {inj }}}\right) c_{2}^{\text {inj }} F\left(\lambda_{n}\right)\left(\lambda_{3}-\gamma_{2}\left(s, \lambda_{n}\right)\right)\left(\lambda_{4}-\gamma_{2}\left(s, \lambda_{n}\right)\right)}{\psi_{3}(s)\left(\lambda_{3}-\lambda_{4}\right) s}\left[\nu_{1}\left(m_{1} e^{m_{2} x}-m_{2} e^{m_{1}(x-1)+m_{2}}\right)\right] \\
& -\frac{\left(1-e^{-s \tau_{\mathrm{inj}}}\right) c_{2}^{\mathrm{inj}} F\left(\lambda_{n}\right)\left(\lambda_{3}-\gamma_{2}\left(s, \lambda_{n}\right)\right)\left(\lambda_{4}-\gamma_{2}\left(s, \lambda_{n}\right)\right)}{\psi_{3}(s)\left(\lambda_{3}-\lambda_{4}\right) s}\left[v_{2}\left(n_{1} e^{n_{2} x}-n_{2} e^{n_{1}(x-1)+n_{2}}\right)\right],
\end{aligned}
$$




$$
\begin{aligned}
\bar{c}_{H, 2}\left(\lambda_{n}, x, s\right) \\
=\frac{\left(1-e^{-s \tau_{\mathrm{inj}}}\right) \psi_{3}(s) c_{1}^{\mathrm{inj}} F\left(\lambda_{n}\right)}{\left(\lambda_{3}-\lambda_{4}\right) s}\left[\nu_{1}\left(m_{1} e^{m_{2} x}-m_{2} e^{m_{1}(x-1)+m_{2}}\right)\right] \\
\quad-\frac{\left(1-e^{-s \tau_{\mathrm{inj}}}\right) \psi_{3}(s) c_{1}^{\mathrm{inj}} F\left(\lambda_{n}\right)}{\left(\lambda_{3}-\lambda_{4}\right) s}\left[\nu_{2}\left(n_{1} e^{n_{2} x}-n_{2} e^{n_{1}(x-1)+n_{2}}\right)\right] \\
\quad+\frac{\left(1-e^{-s \tau_{\mathrm{inj}}}\right) c_{2}^{\mathrm{inj}} F\left(\lambda_{n}\right)}{\left(\lambda_{3}-\lambda_{4}\right) s}\left[\nu_{1}\left(\lambda_{4}-\gamma_{2}\left(s, \lambda_{n}\right)\right)\left(m_{2} e^{m_{1}(x-1)+m_{2}}-m_{1} e^{m_{2} x}\right)\right] \\
\quad+\frac{\left(1-e^{-s \tau_{\mathrm{inj}}}\right) c_{2}^{\mathrm{inj}} F\left(\lambda_{n}\right)}{\left(\lambda_{3}-\lambda_{4}\right) s}\left[\nu_{2}\left(\lambda_{3}-\gamma_{2}\left(s, \lambda_{n}\right)\right)\left(n_{1} e^{n_{2} x}-n_{2} e^{n_{1}(x-1)+n_{2}}\right)\right] .
\end{aligned}
$$

Here,

$v_{1}=\frac{P e_{z}}{m_{1}\left(P e_{z}-m_{2}\right)+m_{2}\left(m_{1}-P e_{z}\right) e^{m_{2}-m_{1}}}$,

$$
\nu_{2}=\frac{P e_{z, T}}{n_{1}\left(P e_{z, T}-n_{2}\right)+n_{2}\left(n_{1}-P e_{z, T}\right) e^{n_{2}-n_{1}}} .
$$

To get back solutions in the actual coordinates, we again apply the numerical Laplace and Hankel inversions to the above equations (Durbin 1974) as discussed in the Appendix 1 of this manuscript.

\section{Numerical case studies}

Here, a few test problems are carried out to validate the derived analytical solutions. A high resolution finite volume scheme (HR-FVS) of Koren is also applied to the linear and nonlinear models for identifying the validity ranges of

Table 1 Standard parameters used in the test problems

\begin{tabular}{lll}
\hline Parameter & Symbol & Value \\
\hline Reference Henry's constant & $a^{\text {ref }}$ & 1.0 \\
External porosity & $\epsilon_{b}$ & 0.4 \\
Internal porosity & $\epsilon_{p}$ & 0.333 \\
Density times heat capacity of liquid & $\rho^{L} c_{p}^{L}$ & $4 \mathrm{~kJ} / \mathrm{Kl}$ \\
Axial Peclet numbers & $P e_{z}=P e_{z, T}$ & 600 \\
Radial Peclet numbers & $P e_{\rho}=P e_{\rho, T}$ & 15 \\
Dimensionless constant & $\eta$ & 3.1 \\
Dimensionless constant & $\eta_{T}$ & 28.2 \\
Dimensionless constant & $\zeta$ & 40 \\
Dimensionless constant & $\zeta_{T}$ & 88.6 \\
Injected concentration & $c_{\text {inj }}$ & $1 \mathrm{~mol} / 1$ \\
Injected temperature & $T_{\text {inj }}$ & $300 \mathrm{~K}$ \\
Reference temperature & $T_{\text {ref }}$ & $300 \mathrm{~K}$ \\
Time of injection & $\tau_{\text {inj }}$ & 0.3 \\
\hline
\end{tabular}

analytical results and for getting confidence on them (David et al. 2018; Koren 1993; Javeed et al. 2011). The suggested 2D numerical scheme has already been applied and derived in our previous article for isothermal 2D-GRM (David et al. 2018). The derivation of this scheme for the current nonisothermal 2D-GRM follows the same procedure and is, therefore, omitted in this manuscript. For that reason, we refer the reader to our article (David et al. 2018) for more details about the numerical scheme. The considered numerical scheme was programmed in $\mathrm{C}$ language for grid points of $100 \times 40 \times 20$. The coupling between the concentration and thermal fronts is analyzed. All parameters used in the simulation, which are typically used in high performance liquid chromatographic (HPLC) applications, are listed in Table 1.

\subsection{Isothermal case $\left(\Delta H_{A}=0 \mathrm{~kJ} / \mathrm{mol}\right)$}

Here, we start with an isothermal case which is a reference case for the non-isothermal behavior. The plots are given for the Danckwerts boundary conditions using the solutions given in Eqs. (74) and (75). The sample is injected through the inner cylindrical region at the column inlet (i.e. at $x=0$ ). The injected temperature $T_{\text {inj }}$, the initial temperature $T_{\text {init }}$ and the reference temperature $T^{\text {ref }}$ are taken the same. The 3D plots in Fig. 1a and c are obtained at the column outlet. While, the 1D plots in Fig. 1b and d are the outlet concentration and temperature profiles averaged over the whole radius of the column. These plots show that no change occurs in the temperature profile when the enthalpy of adsorption is zero (i.e. $\Delta H_{A}=0 \mathrm{~kJ} / \mathrm{mol}$ ). The $1 \mathrm{D}$ plots show a good agreement between the analytical and numerical results for linear isotherm. The 3D graphs show that the radial transfer with the considered radial Peclet number, $P e_{\rho}=15$, is not fast enough to eliminate the radial gradients introduced via injection.

\subsection{Non-isothermal case $\left(\Delta H_{A} \neq 0 \mathrm{~kJ} / \mathrm{mol}\right)$}

Under this subsection, we analyze the effects of enthalpy of adsorption. Figures 2 and 3 show the plots for inner and 

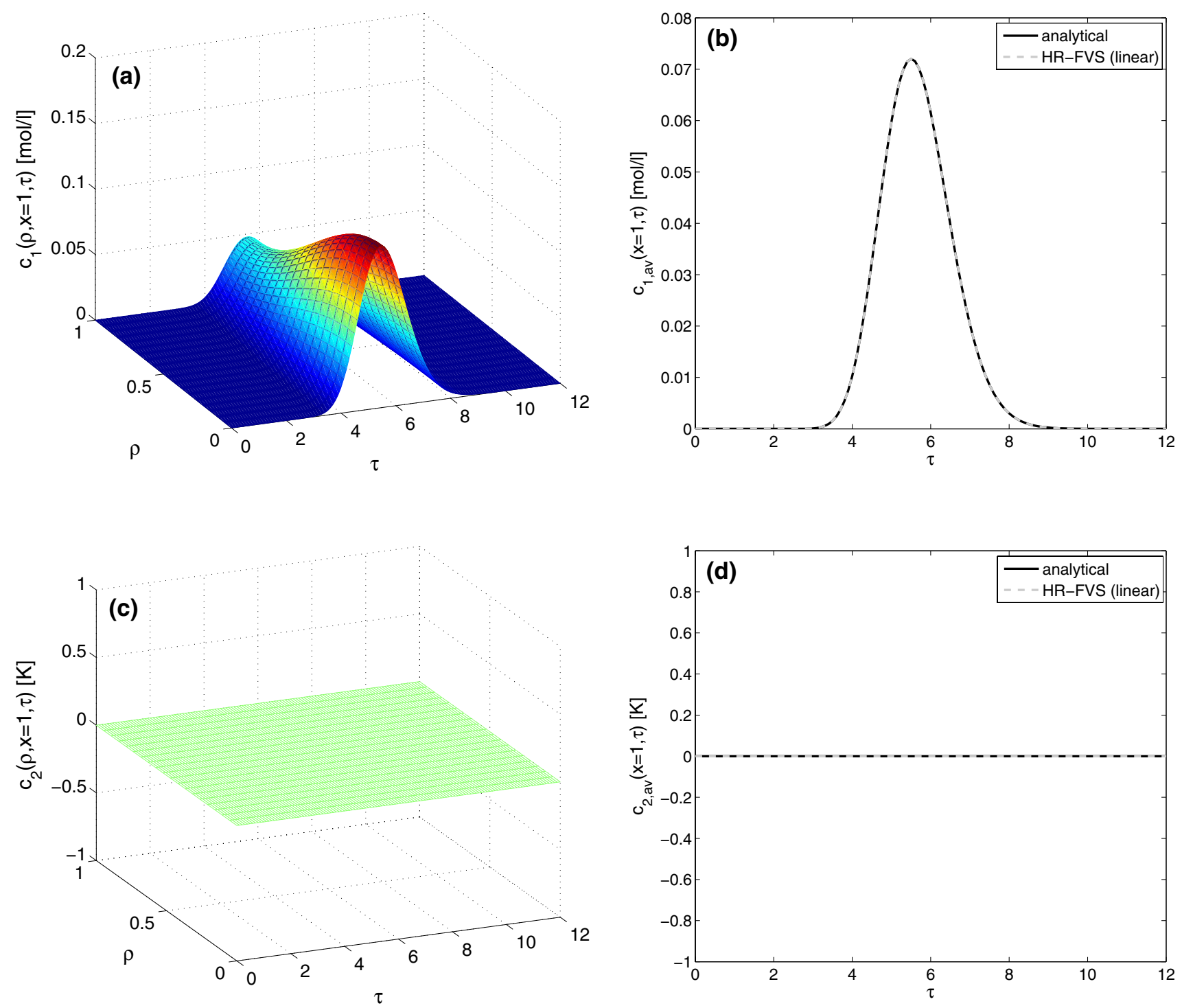

Fig. 1 Isothermal case $\left(\Delta H_{A}=0 \mathrm{~kJ} / \mathrm{mol}\right)$ : Plots a and $\mathbf{c}$ are obtained from analytical solutions, while plots $\mathbf{b}$ and $\mathbf{d}$ show a comparison of analytical and numerical solutions for linear isotherm. Here, Danckwerts boundary conditions and outer zone injection are considered

outer annular zone injections, respectively. The parameters used in both figures are the same and are given in Table 1. Here, we have considered radial Peclet number as $P e_{\rho}=15$ which corresponds to the slow radial dispersion. In the 3D plots at the column outlet, larger values of concentration and temperature profiles can be seen in the inner region of the column for the inner zone injection (c.f. Fig. 2a and c). On the other hand, the concentration and temperature profiles have larger values in the outer region of the column for the outer annular zone injection (c.f. Fig. 3a and c). In Figures $2 b, d$ and $3 b$, $d$, where the averaged concentration and temperature profiles are plotted, it can be seen that a nonzero value of the enthalpy of adsorption $\left(\Delta H_{A}=-10 \mathrm{~kJ} /\right.$ mol) generates fluctuations in the temperature profile without causing any visible change in the concentration profile due to the considered linearization of the isotherm. The plots in Figs. $2 d$ and $3 d$ show that adsorption front elutes first and causes a significant rise in the temperature profile followed by a desorption which causes a drop in the temperature profile. After a complete desorption, the temperature profile attains back its reference value. For the considered two types of injections, a strong similarity is observed in the behavior of the plots. In the 1D plots, a good agreement between the analytical and numerical results for linear isotherm verifies the correctness of the analytical solutions and accuracy of the proposed numerical algorithm.

Figure 4 displays the effects of $\Delta H_{A}$ on the averaged concentration and temperature profiles. The linearized analytical results were obtained for the Dancwerts boundary conditions using the solutions in Eqs. (74) and (75), while the 

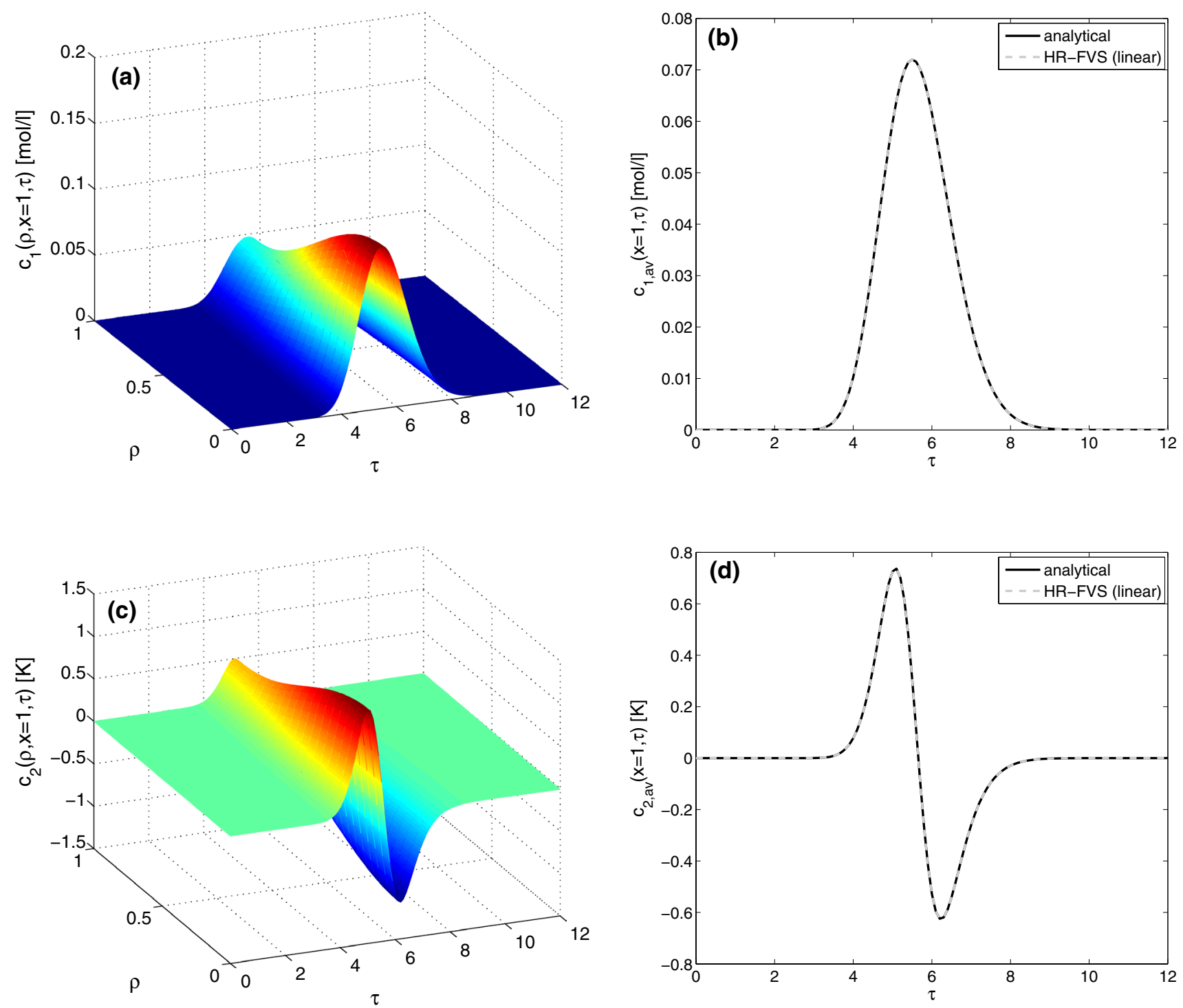

Fig. 2 Non-isothermal case $\left(\Delta H_{A}=-10 \mathrm{~kJ} / \mathrm{mol}\right)$ : Plots $\mathbf{a}$ and $\mathbf{c}$ are obtained from analytical solutions, while plots $\mathbf{b}$ and $\mathbf{d}$ show a comparison of analytical and numerical solutions for linear isotherm. Here, Danckwerts boundary conditions and inner zone injection are considered

numerical solutions were obtained using a nonlinear isotherm given in Eq. (10). It can be seen that linear analytical solutions deviate from the nonlinear solutions of the HRFVS for $\left|\Delta H_{A}\right| \geq 10 \mathrm{~kJ} / \mathrm{mol}$. Moreover, the analytical solutions give over predicted results, while the numerical solutions show accurate deviations from the isothermal behavior for larger values of the enthalpy of adsorption. Thus, the assumption for linearizing the isotherm for moderate adsorption enthalpy of adsorption is justified by these results.

\subsection{Non-isothermal case: effects of dispersions and mass transfer coefficients}

Figures 5 and 6 show the effects of radial Peclet numbers (or radial dispersion coefficients) on the solution profiles for a nonzero enthalpy of adsorption $\left(\Delta H_{A}=-10 \mathrm{~kJ} / \mathrm{mol}\right)$. The plots are once again produced by using Eqs. (74) and (75) for the inner zone injection and keeping all other parameters the same as given in Table 1. It can be seen in Fig. 5a and b that a smaller value of the $P e_{\rho}$ (here 1.5) generates no concentration and temperature gradients along the radial coordinate of the column, i.e. the radial dispersion is faster enough to eliminate the concentration and temperature gradients introduced via injection at the column inlet $(x=0)$. This indicates that smaller values of the radial Peclet number reduce the current 2D-model to a 1D-model, as no dynamics are observed along the radial coordinate of the column. This fact is also verified by the $1 \mathrm{D}$ plots for averaged concentrations in Fig. 5f. On the other hand, for larger value of the $P e_{\rho}$ (here 25 ), dynamics in the concentration and temperature profiles 

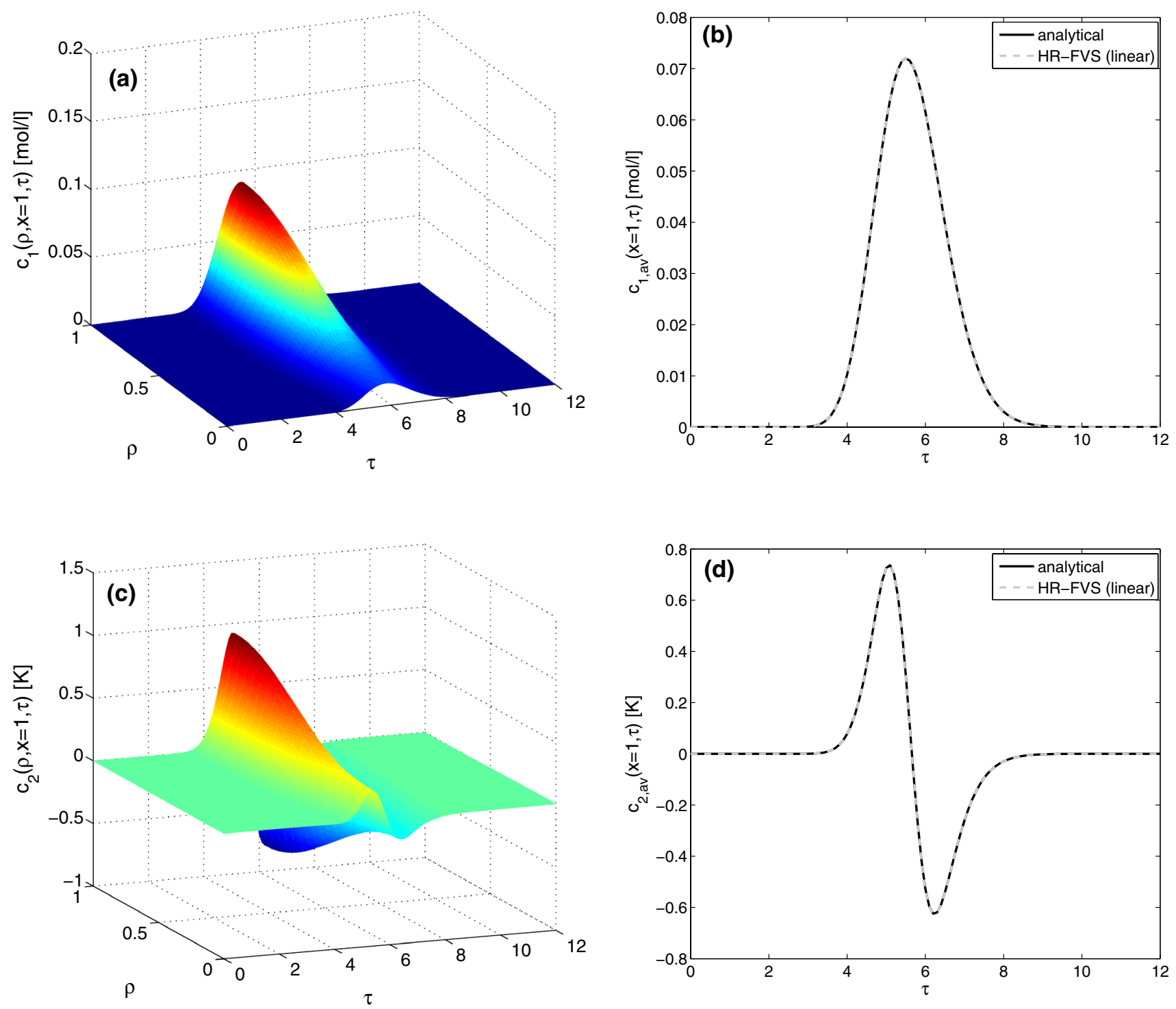

Fig. 3 Non-isothermal case $\left(\Delta H_{A}=-10 \mathrm{~kJ} / \mathrm{mol}\right)$ : Plots $\mathbf{a}$ and $\mathbf{c}$ are obtained from analytical solutions, while plots $\mathbf{b}$ and $\mathbf{d}$ show a comparison of analytical and numerical solutions for linear isotherm. Here, Danckwerts boundary conditions and outer zone injection are considered

are visible along the radial coordinate of the column (c.f. Fig. $5 \mathrm{c}$ and d). This indicates that larger value of $P e_{\rho}$ (i.e. slower radial dispersion) slows down the rate of elimination of concentration gradients introduced during the injection. Furthermore, the 1D plots in Fig. 5e displays the dynamic evolution of radial concentration gradients at the center of the column (i.e. at $x=0.5$ ) which also justifies our above discussion. The concentration plots in Fig. 5f show a comparison of solutions obtained from the 1D-GRM and from the current 2D-GRM. The solutions of 2D-GRM are plotted for three different values of the radial Peclet number (i.e. for $\left.P e_{\rho}=1.5,15,25\right)$ which only appears in this $2 \mathrm{D}$-model. A clearer difference is visible between the solutions of the $1 \mathrm{D}$ and 2D-models for the cases of larger radial Peclet numbers (i.e. for columns of larger diameters) as compared to the case of smaller radial Peclet number (i.e. for a column of smaller diameter). Thus, the application of 2D-model is only useful for the columns having larger radial Peclet numbers. Similar behavior can be observed for the radial Peclet number of temperature $\left(P e_{\rho, T}\right)$ It can be seen in Fig. 6a and c that $P e_{\rho, T}$ has no visible effect on the concentration profiles which is also evident from the 1D plots given in Fig. 6e. The 1D plots are again given at the center of the column (at $x=0.5$ ). It should be noted that while investigating the effects of $P e_{\rho}$ by increasing or reducing its values, the value of $P e_{\rho, T}=15$ was kept fixed and vice-versa. 

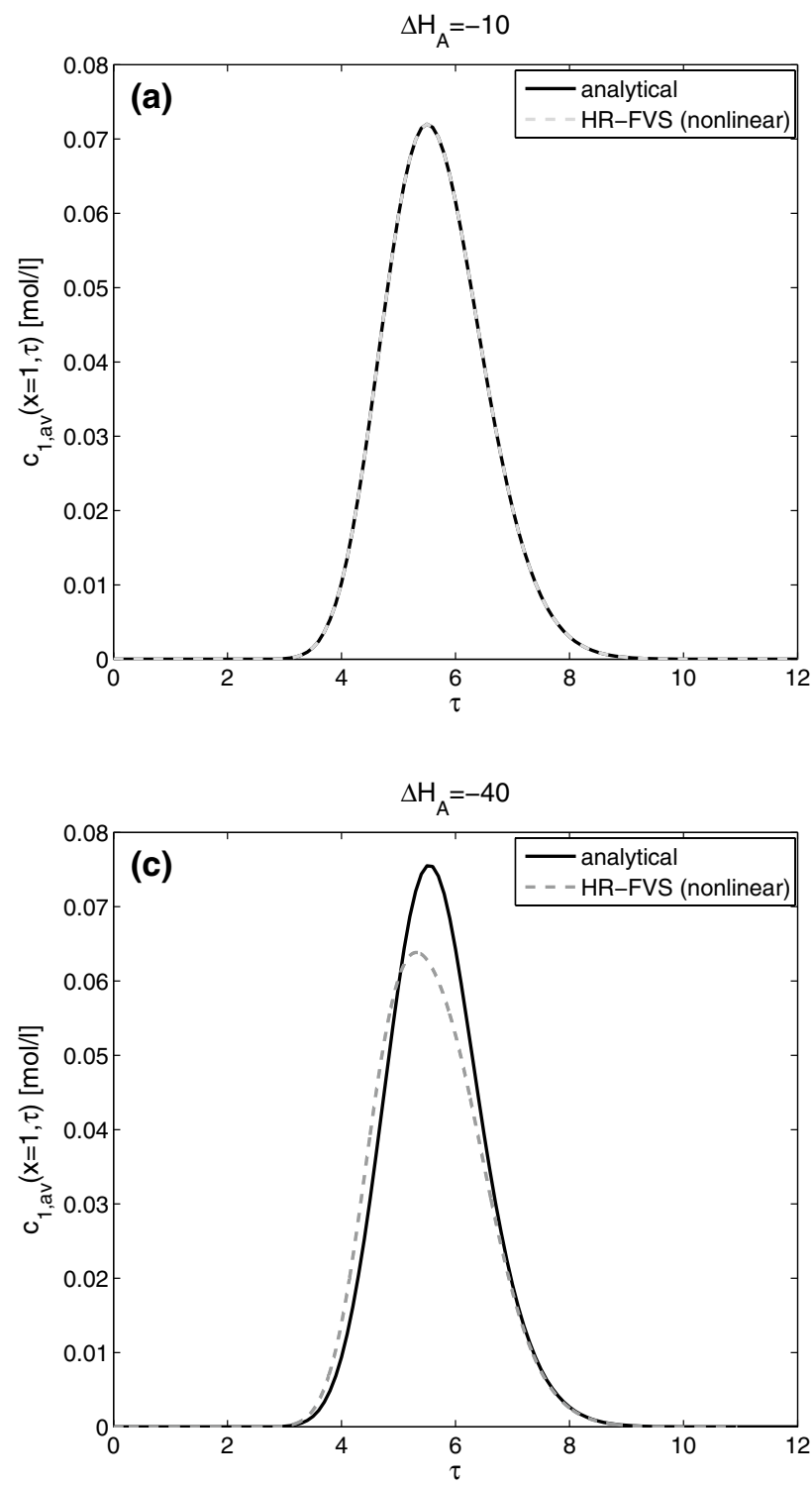

Fig. 4 Non-isothermal case $\left(\Delta H_{A} \neq 0 \mathrm{~kJ} / \mathrm{mol}\right)$ : A comparison of analytical solutions for linear isotherm with the numerical solutions for nonlinear isotherm considering different values of enthalpy of

Figure 7 shows the results for different values of $\mathrm{Pe}_{z}$ and $P e_{z, T}$ while keeping $P e_{\rho}=15=P e_{\rho, T}$ fixed. It is evident that solutions generated by a smaller value of $P e_{z}$ (here 100) are broadened and have lowest peaks as compared to the results generated by higher values of $P e_{z}$ (here 300 and 600).

Figure 8 shows the comparison of analytical solutions for two different boundary conditions (BCs) (i.e. for Danckwerts and Dirichlet BCs). Here, the 1D plots of averaged concentration and temperature (integrated over the radius of the column) are shown. The results were achieved by changing the values of $P e_{z}$ while keep all other parameters fixed as given in Table 1. It can be seen that for a larger axial Peclet number (i.e. smaller axial dispersion) the solutions of both
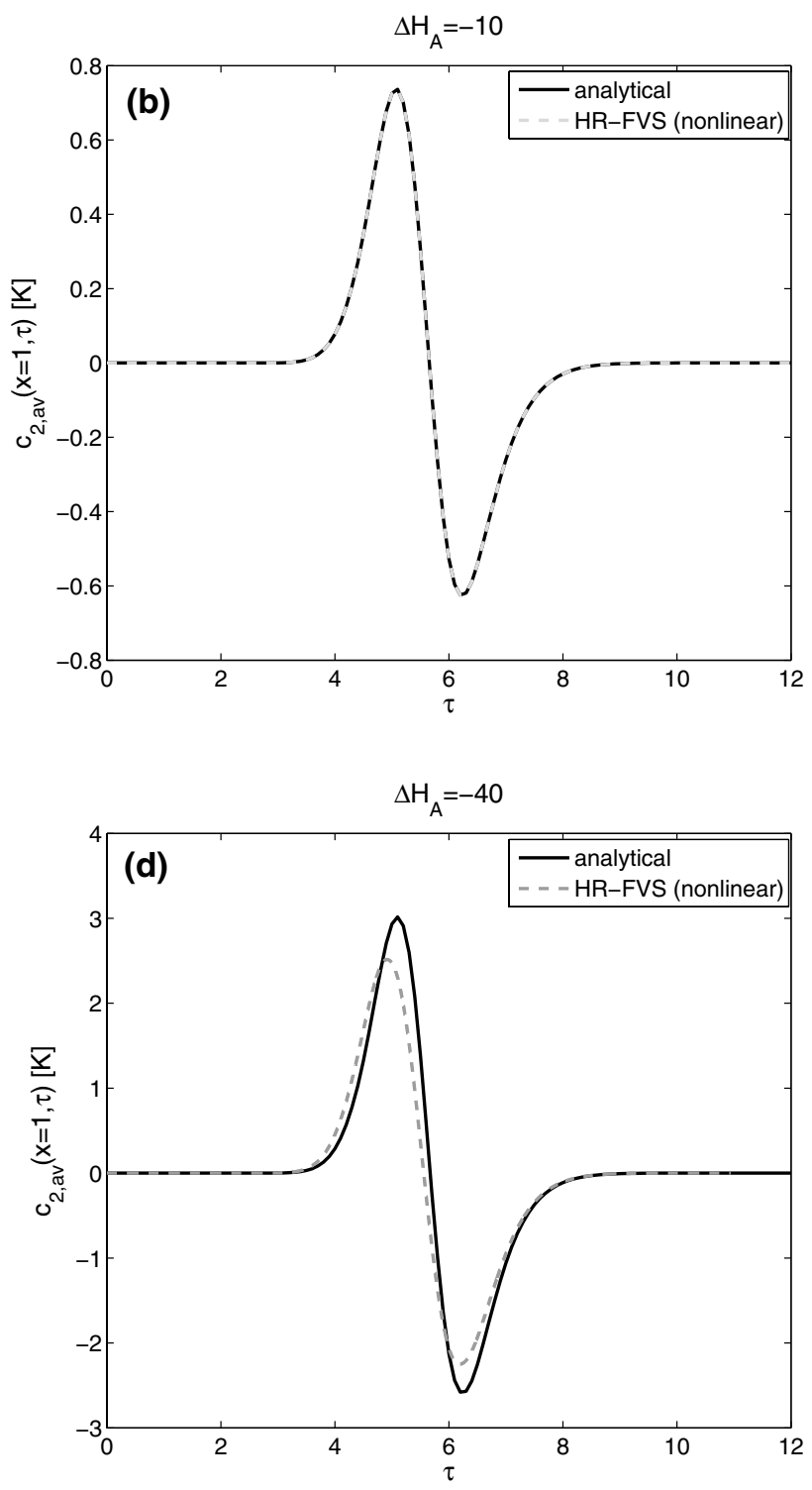

adsorption $\left(\Delta H_{A}\right)$. Here, inner zone injection and Danckwerts boundary conditions are considered

BCs are the same. However, for a smaller axial Peclet number (or larger axial dispersion coefficient), differences can be seen in the concentration and temperature profiles obtained for these two types of BCs. Moreover, the retention times are increased as the axial Peclet numbers are reduced (i.e. the column efficiency is reduced). Except for the concentration profiles, on which $P e_{z, T}$ has no obvious effect, similar results were witnessed when the value of $P e_{z, T}$ was changed while keeping $P e_{z}$ unchanged. Figure 9 shows the effects of model parameter $\eta$, which expresses the intraparticle diffusivity of the fluid, and parameter $\eta_{T}$, which expresses the intraparticle diffusivity of the temperature. We noticed that $\eta_{T}$ has no obvious effects on the averaged concentration 

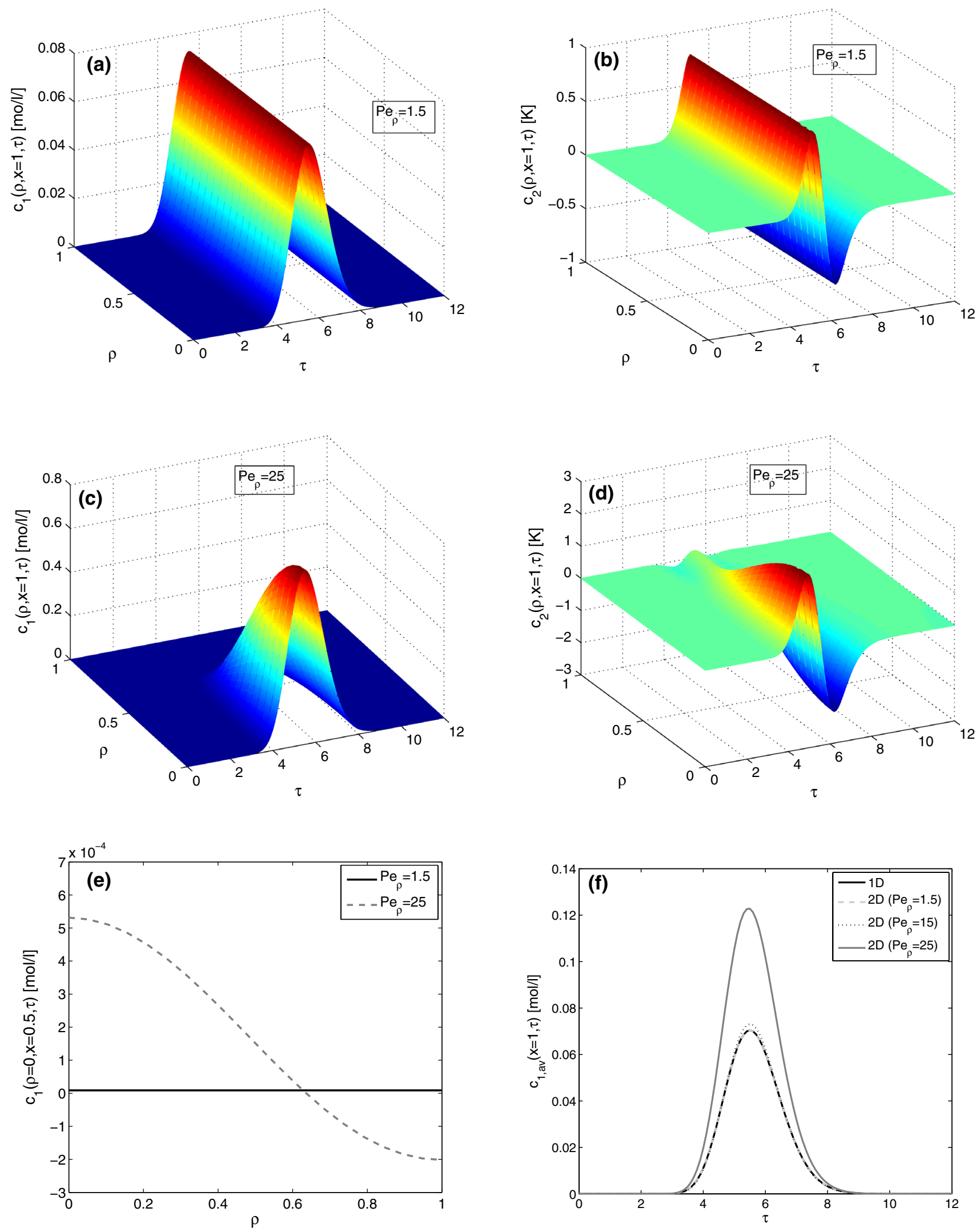

Fig. 5 Non-isothermal case $\left(\Delta H_{A}=-10 \mathrm{~kJ} / \mathrm{mol}\right)$ : Analytical solutions for different values of radial Peclet number $\left(P e_{\rho}\right)$. Plots in e are given at the center of the column $(x=0.5)$ and plots in (f) compare

the solution of 1D-GRM with the solutions of current 2D-GRM. Here, inner zone injection and Danckwerts Boundary conditions are considered 

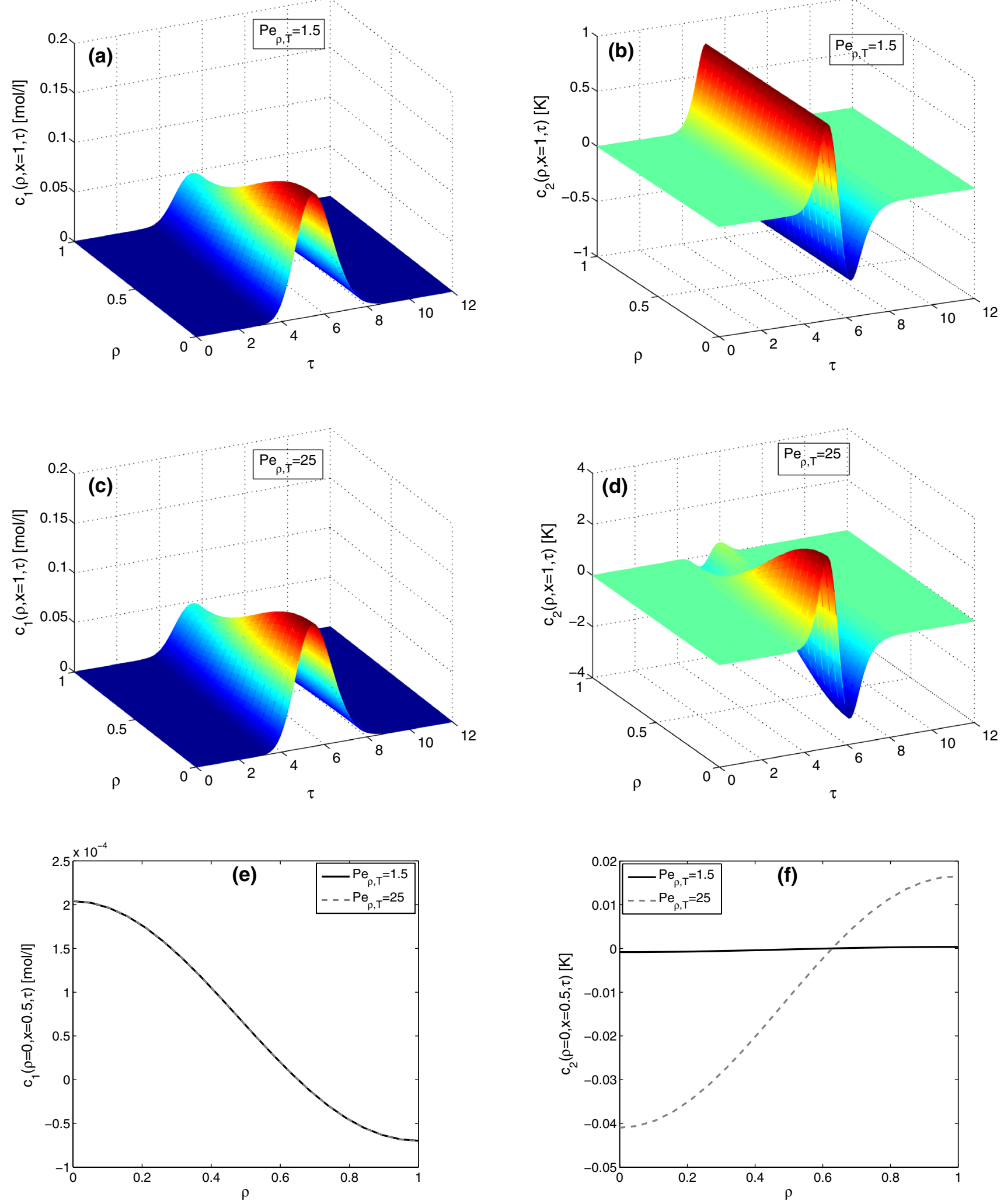

Fig. 6 Non-isothermal case $\left(\Delta H_{A}=-10 \mathrm{~kJ} / \mathrm{mol}\right)$ : Analytical solutions for different values of $P e_{\rho, T}$. Here, plots in e and $\mathbf{f}$ show $1 \mathrm{D}$ plots at the center of the column $(x=0.5)$. Also, inner zone injection and Danckwerts boundary conditions are considered 

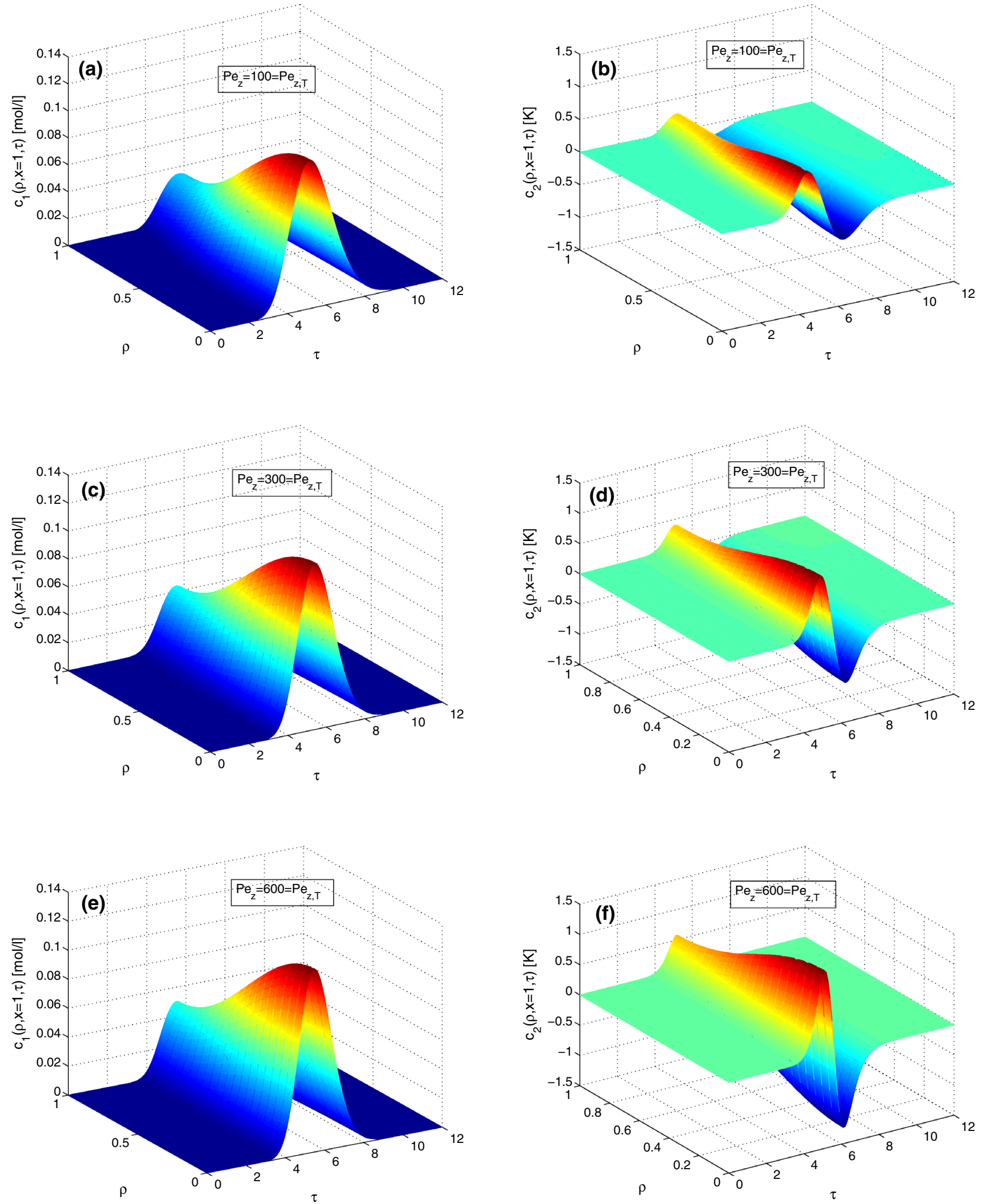

Fig. 7 Non-isothermal case $\left(\Delta H_{A}=-10 \mathrm{~kJ} / \mathrm{mol}\right)$ : Analytical solutions for different values of $P e_{z}$ and $P e_{z, T}$. Here, inner zone injection and Danckwerts boundary conditions are considered 

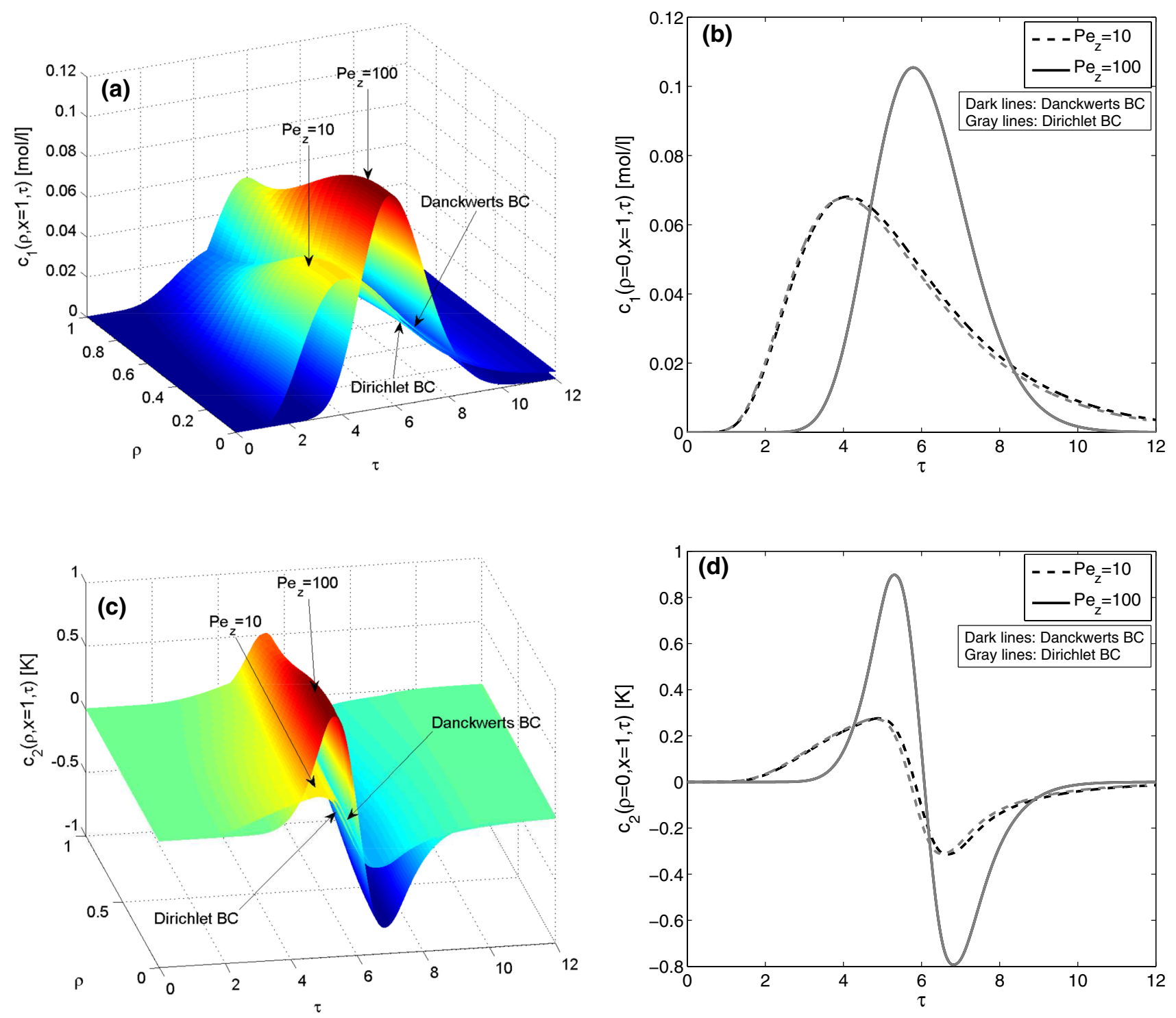

Fig. 8 Non-isothermal case $\left(\Delta H_{A}=-10 \mathrm{~kJ} / \mathrm{mol}\right)$ : A comparison of analytical solutions for Dirichlet and Danckwerts boundary conditions. The effect axial Peclet number $\left(P e_{z}\right)$ (or axial dispersion coef-

profile (Fig. 9c). The diffusion rates are relatively slow for small values of $\eta$ and $\eta_{T}$, which leads to tailed and broadened peaks of the averaged concentration and temperature profiles.

\subsection{Non-isothermal case: effect of the $\frac{\rho^{S} c_{p}^{S}}{\rho^{L} c_{p}^{L}}$}

Here, we analyze the effect of the ratio $\frac{\rho^{s} c_{p}^{s}}{\rho^{L} c_{p}^{L}}$ on the solution profiles under the non-isothermal operation, while all other parameters are kept the same as given in Table 1. In Fig. 10a and $\mathrm{b}$, we have chosen $\rho^{S} c_{p}^{S}=4$ and $\rho^{L} c_{p}^{L}=60$. It is evident

ficient) is analyzed on these solutions. Here, inner zone injection and Danckwerts boundary conditions are considered

that adsorption part of the temperature profile is moving faster and is decoupled from the concentration profile, while the slower desorption part is coupled with the concentration profile. Thus, the temperature wave is moving faster than the concentration profile. In Fig. 10c and d, for $\rho^{S} c_{p}^{S}=4$ and $\rho^{L} c_{p}^{L}=4$, we see that both the concentration and temperature waves are moving at almost the same speed. Lastly, in Fig. 10e and f, for $\rho^{S} c_{p}^{S}=60$ and $\rho^{L} c_{p}^{L}=4$, we can see that the speed of concentration profile is larger than the speed of temperature wave. It is also evident that adsorption part of the temperature wave is coupled with the faster moving concentration profile, while the slower desorption part leaves the column at a later time. 


\subsection{Non-isothermal case: numerical temporal moments of concentration profiles}

Here, the temporal moments of outlet concentration profiles are numerically calculated up to the fourth order. At the column outlet $(x=1)$, the temporal moments along the radial coordinate of the column are obtained as

$\mu_{i}(\rho)=\int_{0}^{\infty} c_{1}(\rho, x=1, \tau) \tau^{i} d \tau, \quad i=1,2,3,4$,

The normalized averaged moments at the column outlet $(x=1)$ are obtained as

$\mu_{i, \mathrm{av}}=\frac{\int_{0}^{\infty} c_{1, \mathrm{av}}(x=1, \tau) \tau^{i} d \tau}{\mu_{0, \mathrm{av}}}, \quad i=1,2,3,4$,
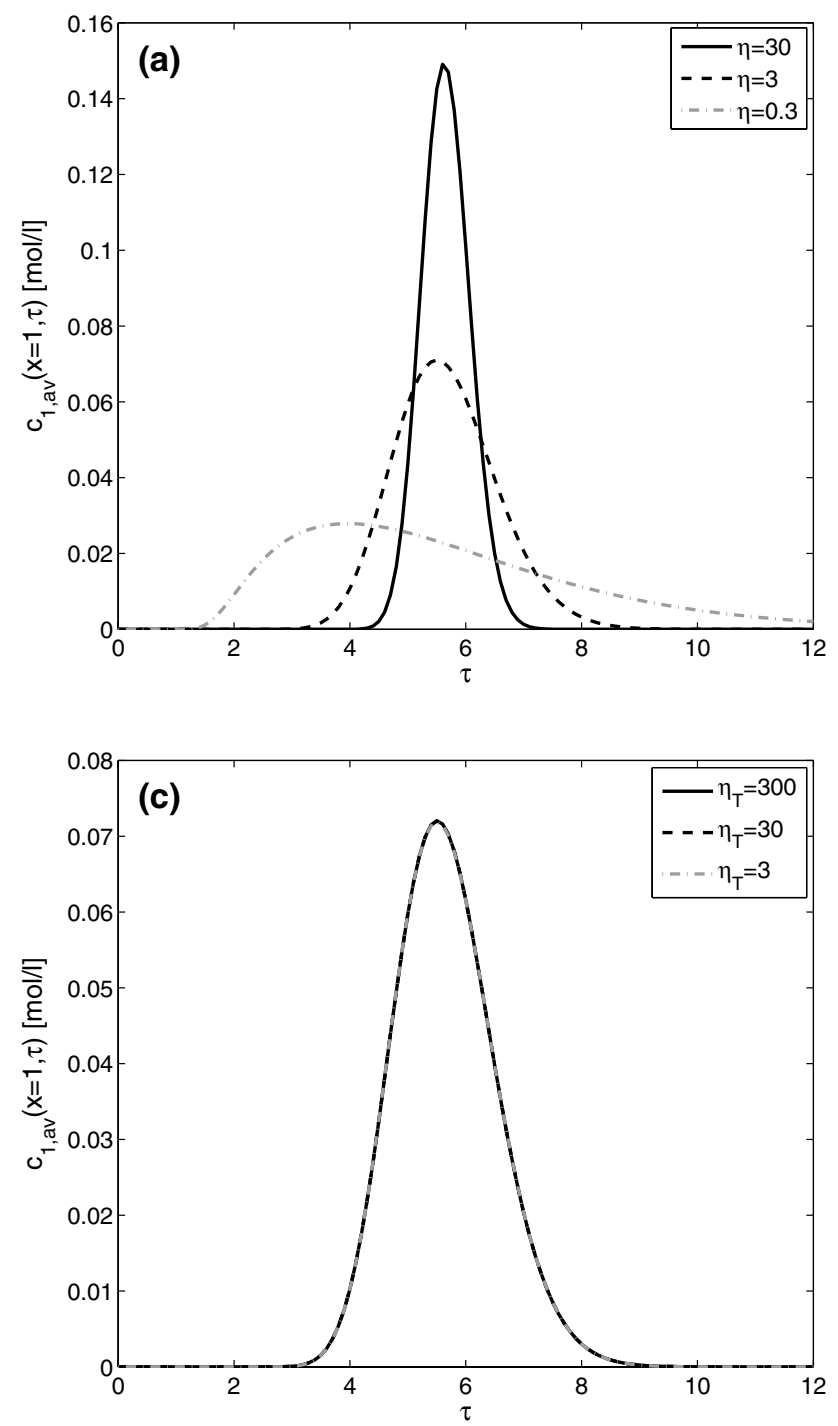

Fig. 10 Non-isothermal case $\left(\Delta H_{t_{c}} \bar{s}-10 \mathrm{~kJ} / \mathrm{mol}\right)$ : Analytical solutions analyzing the effect of ratio $\frac{\rho^{\alpha} c_{p}^{S}}{\rho^{L} c_{p}^{L}}$. Here, inner zone injection and Danckwerts boundary conditions are considered

where, $\mu_{0, \text { av }}$ is given by

$\mu_{0, \mathrm{av}}=\int_{0}^{\infty} c_{1, \mathrm{av}}(x=1, \tau) d \tau$

and the averaged concentration $c_{1, \mathrm{av}}$ is obtained by integration $c_{1}(\rho, x=1, \tau)$ over $\rho$ :

$c_{1, \mathrm{av}}(x=1, \tau)=2 \int_{0}^{1} c_{1}(\rho, x=1, \tau) \rho d \rho$.
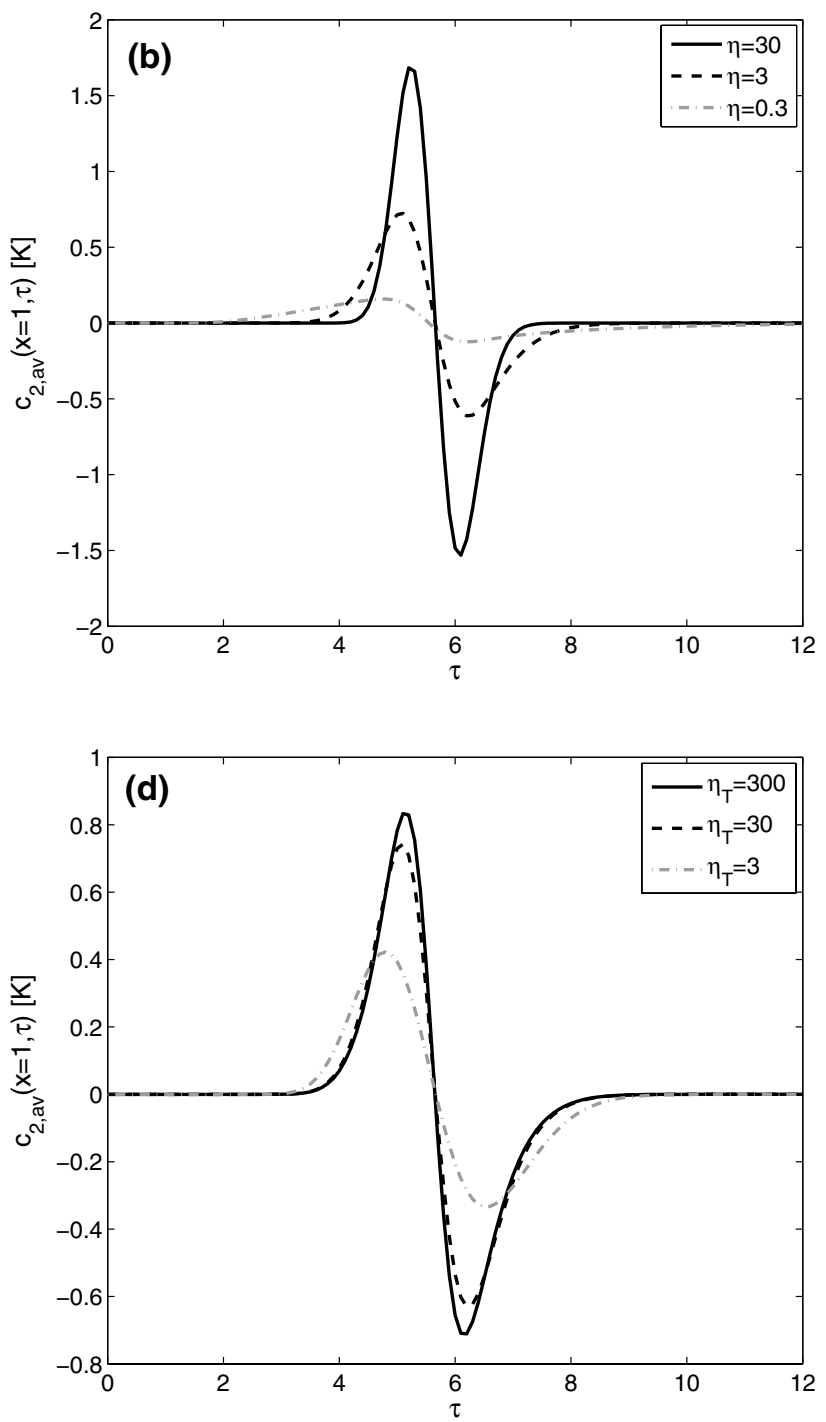

Fig. 9 Non-isothermal case $\left(\Delta H_{A}=-10 \mathrm{~kJ} / \mathrm{mol}\right)$ : Analytical solutions analyzing the effects of $\eta$ and $\eta_{T}$. Here, inner zone injection and Danckwerts boundary conditions are considered 

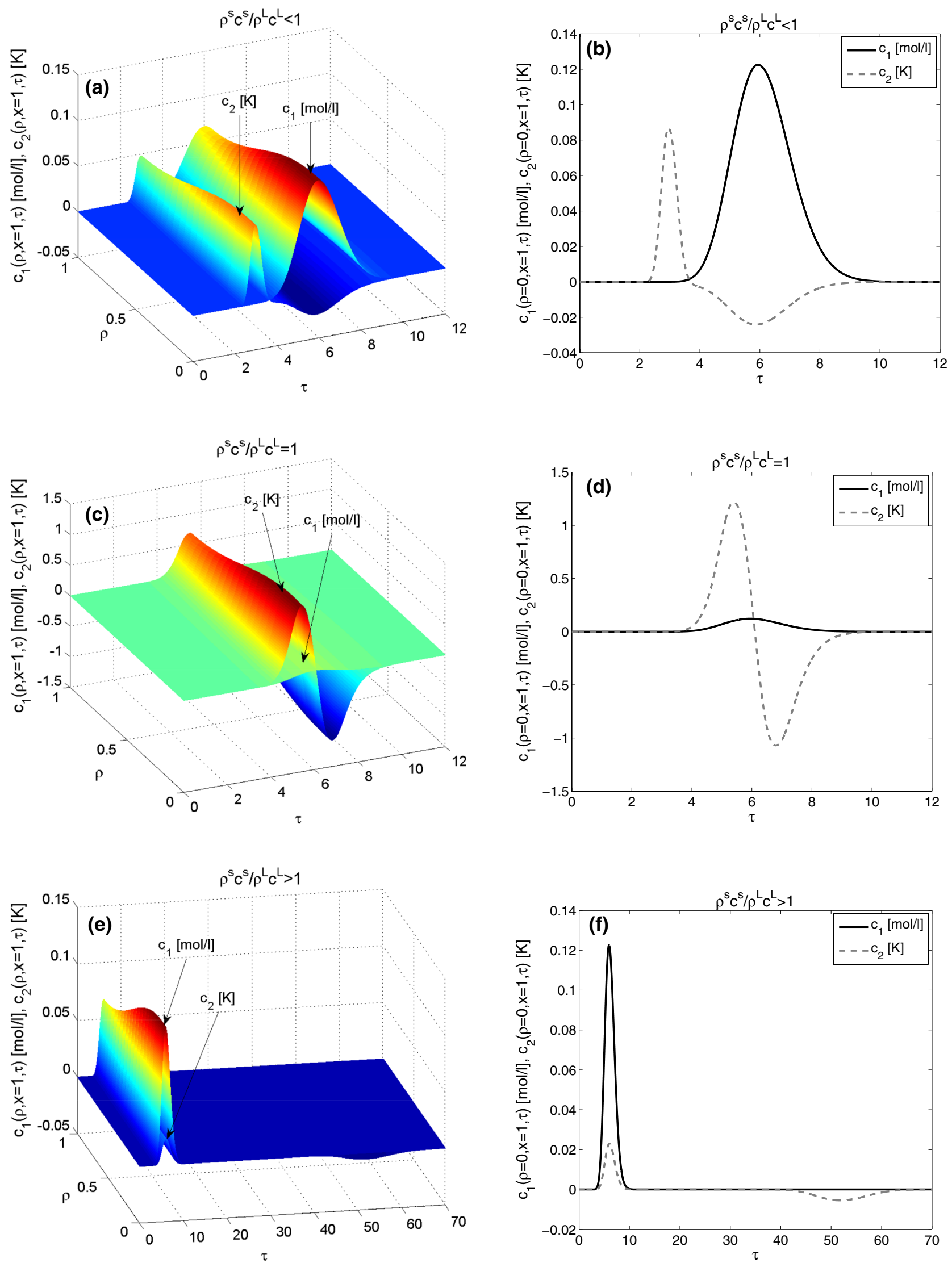
Furthermore, the normalized averaged central moment is calculated by

$\mu_{i, \mathrm{av}}^{\prime}=\frac{\int_{0}^{\infty} c_{1, \mathrm{av}}(x=1, \tau)\left(\tau-\mu_{1, \mathrm{av}}\right)^{i} d \tau}{\mu_{0, \mathrm{av}}}, \quad i=2,3,4$

The dimensionless moments are first plotted to analyze the effects of axial dispersion coefficient $\left(P e_{z}\right)$ on the concentration profile using the solution of the Dirichlet BCs for injections via the inner zone. The Dancwerts BCs give similar results. Figure 11 displays the plots of averaged central moments for the concentration profile. It can be seen that
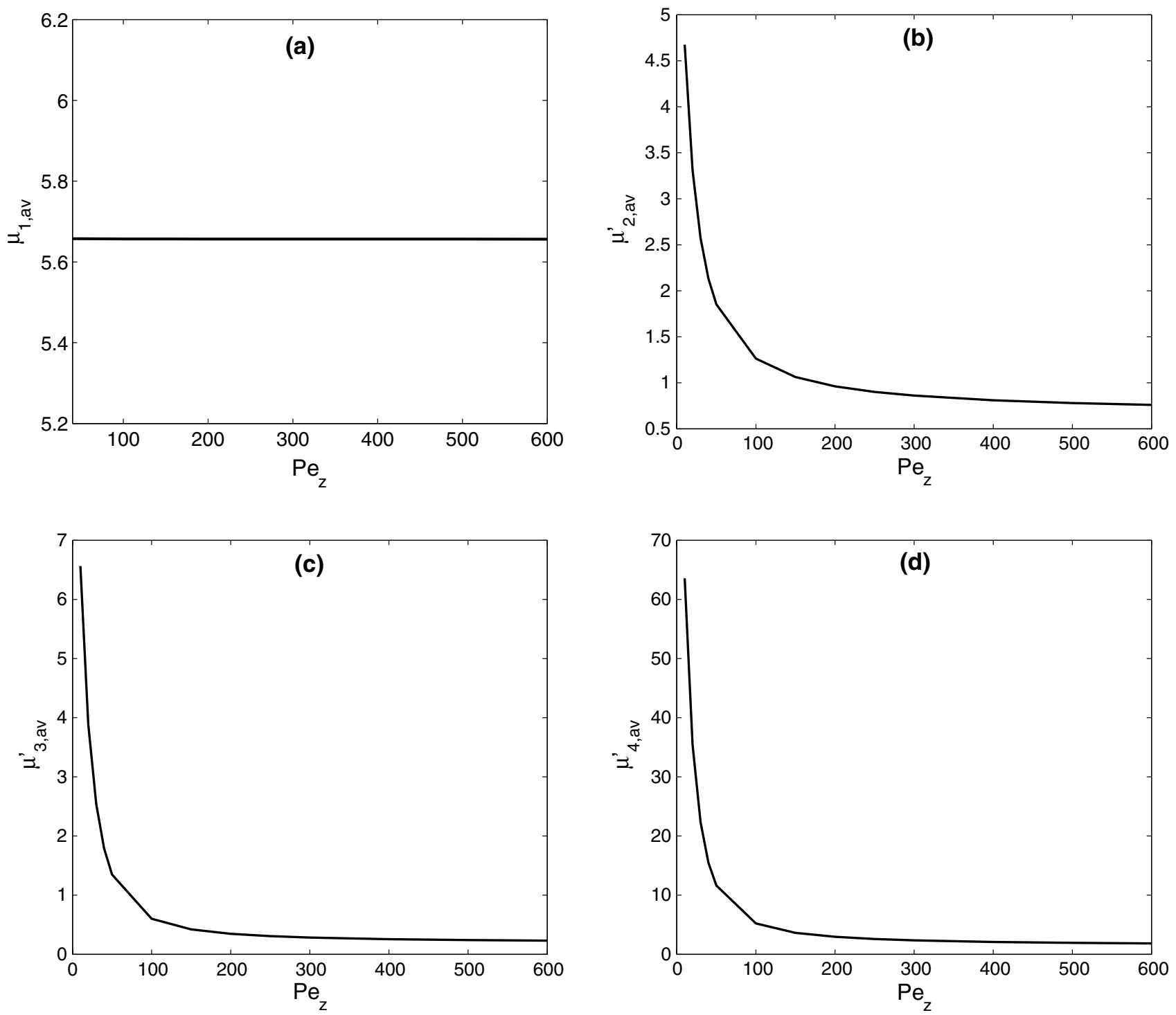

$P e_{z}$ has no effects on the first moments. However, it affects the second, third and fourth moments. As the value of $P e_{z}$ increases, the values of moments decreases. These results also agree with those presented in Fig. 8 .

Figure 12 illustrates the effects of $P e_{\rho}$ on the local moments obtained through Eq. (78). These plots indicate that smaller values of $P e_{\rho}$ generate constant values of the moments along the radial coordinate $(\rho)$ of the column. In the case of outer zone injection, similar results were recorded. These results also agree well with those shown in Fig. 5.

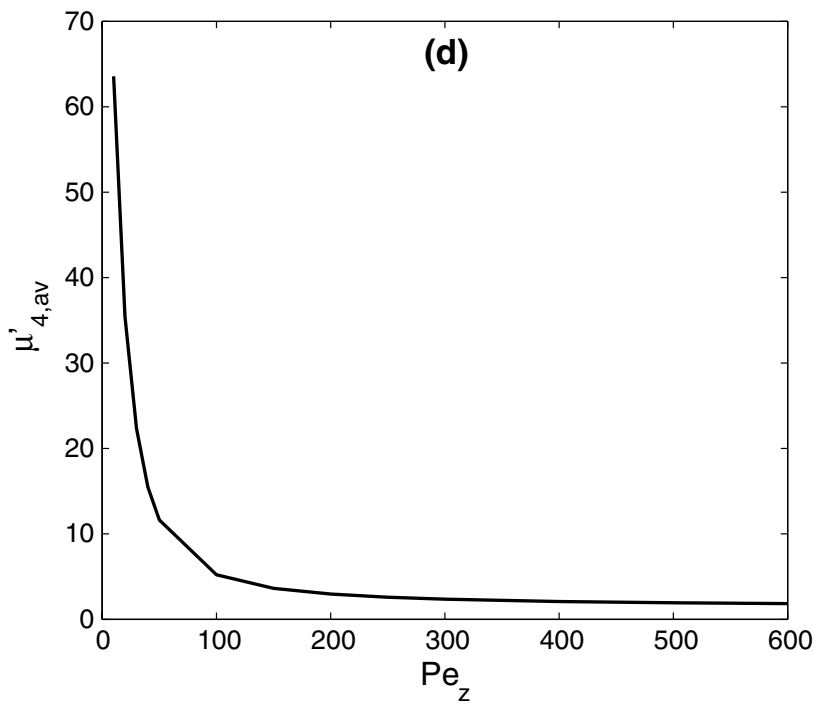

Fig. 11 Non-isothermal case $\left(\Delta H_{A}=-10 \mathrm{~kJ} / \mathrm{mol}\right)$ : The first four averaged temporal moments of $c_{1}[\mathrm{~mol} / l]$ for Dirichlet boundary conditions. Here, inner zone injection is considered 

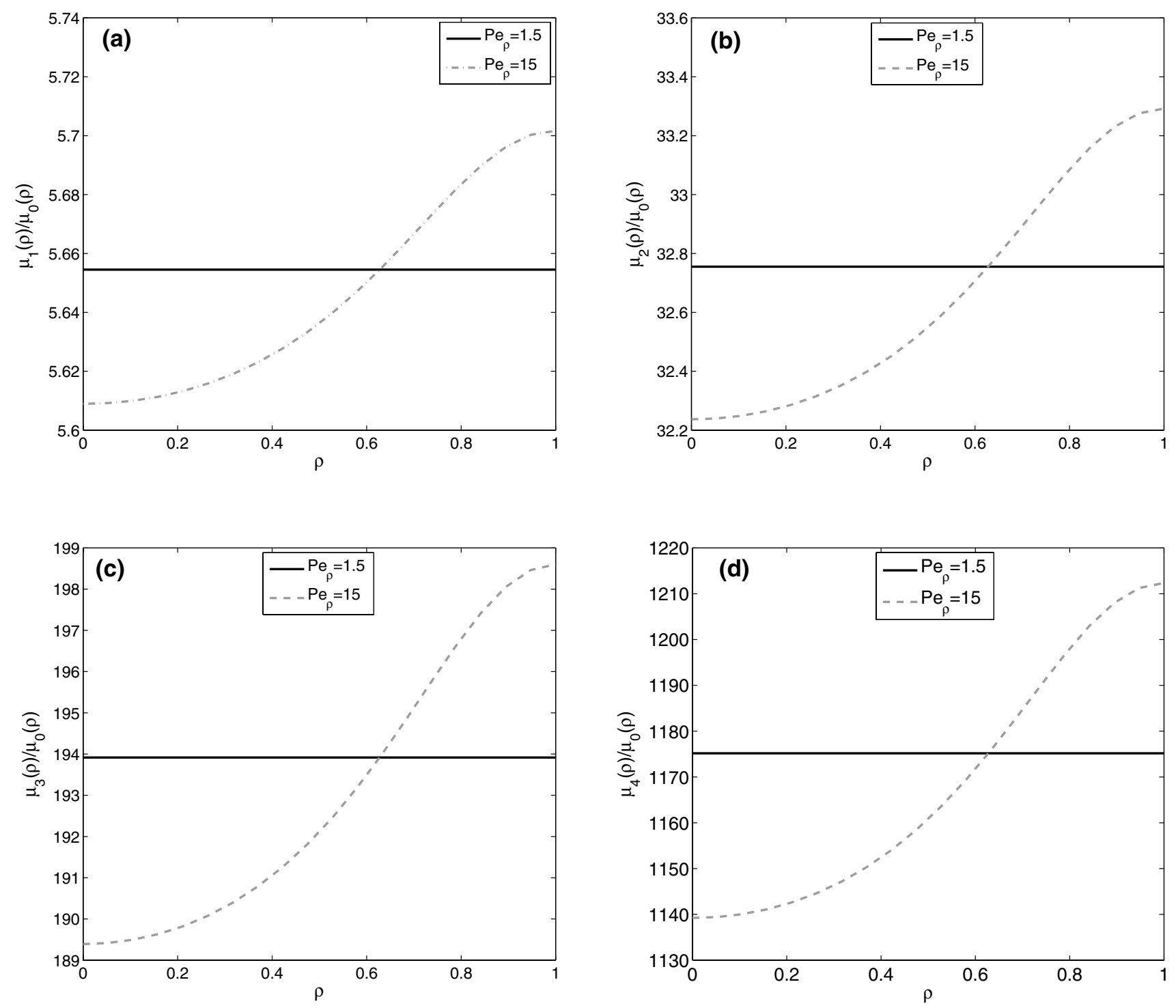

Fig. 12 Non-isothermal case $\left(\Delta H_{A}=-10 \mathrm{~kJ} / \mathrm{mol}\right)$ : The effect of radial Peclet number $\left(P e_{\rho}\right)$ is analyzed on the local moments. Here, inner zone injection and Dirichlet boundary conditions are considered

\section{Conclusion}

A linearized non-isothermal two-dimensional general rate model was formulated and analytically solved to investigate the effects of temperature fluctuations in liquid chromatographic columns of cylindrical geometry. The analytical solutions were derived by successively applying the finite Hankel and Laplace transformations along with the eigendecomposition technique and a conventional solution technique of ordinary differential equations (ODEs). The solutions were derived for Dirichlet and Dancwerts boundary conditions. In order to verify the analytical results and to find their validity ranges, a high resolution finite volume scheme was applied to numerically approximate the model equations for linear and nonlinear isotherms. Several case studies, covering a wide range of kinetic and thermodynamic parameters, were conducted to demonstrate the coupling between the concentration and thermal waves. The derived analytical solutions of the 2D-model can play significant roles in further developments of the non-isothermal chromatography. For instance, the derived analytical solutions can be used as an effective tool for parameter estimation, for sensitivity analysis and for analyzing the performance of numerical schemes.

Acknowledgements Open access funding provided by Max Planck Society. The first author is grateful to The World Academy of Sciences (TWAS) and COMSATS University Islmabad for the Award of 2015 CIIT-TWAS full time postgraduate fellowship (FR No: 3240287173). 
Open Access This article is distributed under the terms of the Creative Commons Attribution 4.0 International License (http://creativecommons.org/licenses/by/4.0/), which permits unrestricted use, distribution, and reproduction in any medium, provided you give appropriate credit to the original author(s) and the source, provide a link to the Creative Commons license, and indicate if changes were made.

\section{Appendix 1}

\section{Numerical Laplace inversion technique}

To achieve back the time domain solution $c_{H, j}\left(\lambda_{n}, x, \tau\right)$, for $j=1,2$, we follow the following numerical procedure, which is based on a Fourier series expansion (Durbin 1974; Rice and Do 1995). The analytical Laplace inversion formula is given as

$$
\begin{aligned}
c_{H, j}\left(\lambda_{n}, x, \tau\right) & =\mathcal{L}^{-1}\left[\bar{c}_{H, j}\left(\lambda_{n}, x, s\right)\right] \\
& =\frac{1}{2 \pi i} \int_{a-i \infty}^{a+i \infty} e^{-\tau s} \bar{c}_{H, j}\left(\lambda_{n}, x, s\right) d s, \quad j=1,2,
\end{aligned}
$$

with $s=a+i b ; a, b \in \mathbb{R}$ and integration is taken along the Bromwich contour. Therefore, the real part of all the singularities of $\bar{c}_{H, j}\left(\lambda_{n}, x, s\right)$ is exceeded by the real constant $a$. The integrals in Eqs. (28) and (A-1) exist for $\operatorname{Re}(s)>\gamma \in \mathbb{R}$ if

(a) $c_{H, j}$ is locally integrable,

(b) there is a $\tau_{0} \geq 0$ and $p, \gamma \in \mathbb{R}$, such that $c_{H, j}\left(\lambda_{n}, x, \tau\right) \leq p e^{\gamma \tau}$ for all $\tau \geq \tau_{0}$,

(c) for all $\tau \in(0, \infty)$, there exists a neighborhood in which $c_{H, j}$ is of bounded variation.

In the discussion below, it is assumed always that $c_{H, j}$ fulfils the aforementioned conditions and additionally, no singularities of $\bar{c}_{H, j}\left(\lambda_{n}, x, s\right)$ exist on the right side of the origin. Thus, Eqs. (28) and (A-1) are well-defined for all $y>0$. The provision for selecting $a>0$ arbitrarily provides a foundation for the Durbin methods Durbin (1974). The integral in Eq. (A-1) is given in the interval $[0,2 T]$ equivalently as Durbin (1974)

$$
\begin{aligned}
c_{H, j}\left(\lambda_{n}, x, \tau\right)= & \frac{e^{a \tau}}{\pi} \int_{0}^{\infty}\left[\operatorname{Re}\left\{\bar{c}_{H, j}\left(\lambda_{n}, x, s\right)\right\} \cos (b \tau)\right. \\
& \left.-\operatorname{Im}\left\{\bar{c}_{H, j}\left(\lambda_{n}, x, s\right)\right\} \sin (b \tau)\right] d b .
\end{aligned}
$$

Finally, the following approximate expression is used to replace the expression in Eq. (A-2) Durbin (1974):

$$
\begin{aligned}
c_{H, j}\left(\lambda_{n}, x, \tau\right) & \\
= & \frac{e^{a \tau}}{T}\left[-\frac{1}{2} \operatorname{Re}\left\{\bar{c}_{H, j}\left(\lambda_{n}, x, a\right)\right\}\right. \\
& +\sum_{p=0}^{\infty} \operatorname{Re}\left\{\bar{c}_{H, j}\left(\lambda_{n}, x, a+i \frac{p \pi}{T}\right)\right\} \cos \left(\frac{p \pi \tau}{T}\right) \\
& \left.-\sum_{p=0}^{\infty} \operatorname{Im}\left\{\bar{c}_{H, j}\left(\lambda_{n}, x, a+i \frac{p \pi}{T}\right)\right\} \sin \left(\frac{p \pi \tau}{T}\right)\right] .
\end{aligned}
$$

During the computer implementation, the infinite series in Eq. (A-3) have to be replaced by finite number $N_{p}$ of terms only. Therefore, a truncation error appears in the numerical results. In our simulations, we have chosen $N_{p}=10^{3}$.

Hankel inversion technique

Lastly, the following Hankel inversion formula is used to obtain the solution back to the actual radial coordinate Chen et al. (2011):

$$
\begin{aligned}
c_{j}(\rho, x, \tau)= & 2 c_{H, j}\left(\lambda_{n}=0, x, \tau\right) \\
& +2 \sum_{n=1}^{\infty} c_{H, j}\left(\lambda_{n}, x, \tau\right) \frac{J_{0}\left(\lambda_{n} \rho\right)}{\left|J_{0}\left(\lambda_{n}\right)\right|^{2}} .
\end{aligned}
$$

The above solution is again a series solution. In the numerical computations, the infinite series in Eq. (A-4) is added up to a finite number $N_{n}$ of terms only. In our computations, we have used $N_{n}=10^{3}$.

The above techniques were implemented in the Matlab software.

\section{References}

Bellot, J.C., Condoretm, J.S.: Liquid chromatography modelling : a review. Proc. Biochem. 26, 363-376 (1991)

Carslaw, H.S., Jaeger, J.C.: Operational Methods in Applied Mathematics. Oxford University Press, Oxford (1953)

Carta, G.: Exact analytical solution of a mathematical model for chromatographic operations. Chem. Eng. Sci. 43, 2877-2883 (1988)

Cerro, R.L., Smith, J.M.: Effects of heat realse and nonlinear equilibrium on transient adsorption. Ind. Eng. Chem. Fundam. 8, 796-802 (1969)

Chen, J.-S., Liu, Y.-H., Liang, C.-P., Liu, C.-W., Lin, C.-W.: Exact analytical solutions for two-dimensional advection-dispersion equation in cylindrical coordinates subject to third-type inlet boundary conditions. Adv. Water Resour. 34, 365-374 (2011)

Crank, J.: The Mathematics of Diffusion, 2nd edn. Clarendon Press, Oxford (1975)

David, U.U., Qamar, S., Seidel-Morgenstern, A.: Analytical and numerical solutions of two-dimensional general rate models for liquid chromatographic columns packed with core-shell particles. Chem. Eng. Res. Des. 130, 295-320 (2018)

Durbin, F.: Numerical inversion of laplace transforms: an efficient improvement to Dubner and Abate's method. Comput. J. 17, 371-376 (1974) 
Guiochon, G.: Preparative liquid chromatography. J. Chromatogr. A 965, 129-161 (2002)

Guiochon, G., Lin, B.: Modeling for Preparative Chromatography. Academic Press, San Diego (2003)

Guiochon, G., Felinger, A., Shirazi, D.G., Katti, A.M.: Fundamentals of Preparative and Nonlinear Chromatography, 2nd edn. ELsevier Academic press, New York (2006)

Haynes Jr., H.W.: An analysis of sorption heat effects in the pulse gas chromatography diffusion experiment. AIChE J. 32, 1750-1753 (1986)

Javeed, S., Qamar, S., Seidel-Morgenstern, A., Warnecke, G.: Efficient and accurate numerical simulation of nonlinear chromatographic processes. J. Comput. Chem. Eng. 35, 2294-2305 (2011)

Javeed, S., Qamar, S., Seidel-Morgenstern, A., Warnecke, G.: Parametric study of thermal effects in reactive liquid chromatography. Chem. Eng. J. 191, 426-440 (2012)

Koren, B.: A robust upwind discretization method for advection, diffusion and source terms. In: Vreugdenhil, C.B., Koren, B. (eds.) Numerical Methods for Advection-Diffusion Problems. Notes on Numerical Fluid Mechanics, Chapter 5, vol. 45, pp. 117-138. Vieweg Verlag, Braunschweig (1993)

Leweke, S., von Lieres, E.: Fast arbitrary order moments and arbitrary precision solution of the general rate model of column liquid chromatography with linear isotherm. J. Comput. Chem. Eng. 84, 350-362 (2016)

Miyabe, K.: Moment analysis of chromatographic behavior in reversedphase liquid chromatography. J. Sep. Sci. 32, 757-770 (2009)

Parveen, S., Qamar, S., Seidel-Morgenstern, A.: Analysis of twodimensional non-quilibrium model of linear reactive chromatography considering irreversible and reversible reactions. Ind. Eng. Chem. Res. 55, 2471-2482 (2016)

Qamar, S., Khan, F.U., Mehmood, Y., Seidel-Morgenstern, A.: Analytical solution of a two-dimensional model of liquid chromatography including moment analysis. Chem. Eng. Sci. 116, 576-589 (2014)

Qamar, S., Sattar, F.A., Seidel-Morgenstern, A.: Theoretical investigation of thermal effects in non-isothermal non-equilibrium reactive liquid chromatography. Chem. Eng. Res. Des. 115, 145-159 (2016)
Qamar, S., Perveen, S., Seidel-Morgenstern, A.: Numerical approximation of a two-dimensional nonlinear and nonequilibrium model of reactive chromatography. Ind. Eng. Chem. Res. 55, 9003-9014 (2016)

Qamar, S., Sattar, F.A., Batool, I., Seidel-Morgenstern, A.: Theoretical analysis of the influence of forced and inherent temperature fluctuations in an adiabatic chromatographic column. Chem. Eng. Sci. 161, 249-264 (2017)

Qamar, S., Uche, D.U., Khan, F.U., Seidel-Morgenstern, A.: Analysis of linear two-dimensional general rate model for chromatographic columns of cylindrical geometry. J. Chromatogr. A 1496, 92-104 (2017)

Rice, R.G., Do, D.D.: Applied Mathematics and Modeling for Chemical Engineers. Wiley-Interscience, New York (1995)

Ruthven, D.M.: Principles of Adsorption and Adsorption Processes. Wiley-Interscience, New York (1984)

Sainio, T.: Ion-exchange resins as stationary phase in reactive chromatography. Acta Universitatis Lappeenrantaensis 218, Diss. Lappeenranta University of Technology, Finland (2005)

Sainio, T., Kaspereit, M., Kienle, A., Seidel-Morgenstern, A.: Thermal effects in reactive liquid chromatography. Chem. Eng. Sci. 62, 5674-5681 (2007)

Sainio, T., Zhang, L., Seidel-Morgenstern, A.: Adiabatic operation of chromatographic fixed-bed reactors. Chem. Eng. J. 168, 861-871 (2011)

Sneddon, I.H.: The Use of Integral Transforms. McGraw-Hill, New York (1972)

Suzuki, M.: Notes on determining the moments of the impulse response of the basic transformed equations. J. Chem. Eng. Jpn. 6, 540-543 (1973)

$\mathrm{Vu}$, T.D., Seidel-Morgenstern, A.: Quantifying temperature and flow rate effects on the performance of a fixed-bed chromatographic reactor. J. Chromatogr. A 1218, 8097-8109 (2011)

Zhong, G.M., Meunier, F.: Interference and heat effects: moment analysis for two-component chromatography. J. Chromatogr. A 658, 355-360 (1994)

Publisher's Note Springer Nature remains neutral with regard to jurisdictional claims in published maps and institutional affiliations. 